\title{
Long-term effects of wildfire smoke exposure during early-life on the nasal epigenome in rhesus macaques
}

Anthony P. Brown ${ }^{1}$, Lucy Cai ${ }^{1}$, Benjamin I. Laufer ${ }^{2}$, Lisa A. Miller ${ }^{1,3}$, Janine M. LaSalle ${ }^{2}$, Hong $\mathrm{Ji}^{1,3^{*}}$

Affiliations:

${ }^{1}$ California National Primate Research Center, Davis, CA 95616, USA; ${ }^{2}$ Department of Medical

Microbiology and Immunology, MIND Institute, Genome Center, University of California,

Davis, CA 95616, USA. ${ }^{3}$ Department of Anatomy, Physiology and Cell biology, School of

Veterinary Medicine, University of California, Davis, CA 95616, USA

*Corresponding author: Hong Ji, PhD, Department of Anatomy, Physiology and Cell Biology,

School of Veterinary Medicine, California National Primate Research Center, University of California, Davis, CA, USA. Phone: 530-754-0679. Email: hgji@ucdavis.edu

Running title: DNA methylation and gene expression changes associated with early-life exposure to wildfire smoke in rhesus monkeys

Abstract Word Counts: 271; Total Word Count: 5442 


\section{ABSTRACT \\ Background}

Wildfire smoke is responsible for around $20 \%$ of all particulate emissions in the U.S. and affects millions of people worldwide. Children are especially vulnerable, as ambient air pollution exposure during early childhood is associated with reduced lung function. Most studies, however, have focused on the short-term impacts of wildfire smoke exposures. We aimed to identify long-term baseline epigenetic changes associated with early-life exposure to wildfire smoke. We collected nasal epithelium samples for whole genome bisulfite sequencing (WGBS) from two groups of adult female rhesus macaques: one group born just before the 2008 California wildfire season and exposed to wildfire smoke during early-life $(n=8)$, and the other group born in 2009 with no wildfire smoke exposure during early-life $(n=14)$. RNA-sequencing was also performed on a subset of these samples.

\section{Results}

We identified 3370 differentially methylated regions (DMRs) (difference in methylation $\geq 5 \%$ empirical $\mathrm{p}<0.05)$ and 1 differentially expressed gene $($ FLOT2) $(\mathrm{FDR}<0.05$, fold of change $\geq$ 1.2). The DMRs were annotated to genes significantly enriched for synaptogenesis signaling, protein kinase A signaling, and a variety of immune processes, and some DMRs significantly correlated with gene expression differences. DMRs were also significantly enriched within regions of bivalent chromatin (top odds ratio $=1.46$, q-value $<3 \times 10^{-6}$ ) that often silence key developmental genes while keeping them poised for activation in pluripotent cells.

\section{Conclusions}


These data suggest that early-life exposure to wildfire smoke leads to long-term changes in the methylome over genes impacting the nervous and immune systems, but follow-up studies will be required to test whether these changes influence transcription following an immune/respiratory challenge.

Keywords: Wildfire smoke, whole genome bisulfite sequencing, RNA-sequencing, rhesus macaques, early life 


\section{BACKGROUND}

2 According to the National Interagency Fire Center, there were 50,477 wildfires (4.7 million acres

3 burned) in the United States in 2019. In total, 212 million Americans lived in counties affected

4 by wildfires in 2011 (1). These wildfires have contributed to levels of air pollution in the United

5 States that have been linked to premature death (2-5). About $20 \%$ of all fine particulate

6 emissions in the U.S. are from wildfire smoke, while half of all particulate matter less than 2.5

$7 \mu \mathrm{m}$ in diameter $\left(\mathrm{PM}_{2.5}\right)$ in California resulted from wildfires (2). $\mathrm{PM}_{2.5}$ are especially harmful, as

8 these particles are able to penetrate the respiratory system and the lungs (2). Exposure to these

9 particles has been associated with asthma, bronchitis, lung cancer, and cardiovascular disease (3-

10 5). Young children are especially vulnerable to these negative health effects, as studies have

11 linked air pollution exposure in children to reduced lung function $(6,7)$, reduced height-for-age

12 (8), increased blood pressure (9), and an increased risk of developing asthma and eczema (10).

13 Most of these studies, however, focused on the short-term effects of exposures to wildfire smoke

14 or polluted air and none have performed an unbiased assessment of gene pathways impacted by

15 wildfire smoke exposure.

A cohort of rhesus macaques (Macaca mulatta) that were exposed in their first three

17 months of life to a harsh wildfire season in 2008 in California was previously studied to understand some of the long-term effects of wildfire smoke exposure (11). Peripheral blood

19 mononuclear cells (PBMCs) were cultured and challenged with either LPS or flagellin, and secretions of IL-8 and IL-6 were compared to macaques that were born in $2009\left(\mathrm{PM}_{2.5}\right.$ and ozone

21 levels were much lower in 2009 compared to 2008) (11). Lung function was also compared

22 between exposed and control macaques. Compared to control macaques, wildfire smoke-exposed

23 macaques had significantly reduced lung volume. Female wildfire-exposed macaques showed 
24 reduced production of IL-8 compared to controls, while male wildfire-exposed macaques showed

25 reduced production of IL-6 compared to controls (11). This study implied that early-life exposure

26 led to a difference in IL-8 and IL-6 production following an immune challenge, but it was still

27 unclear to what degree these macaques exhibited baseline differences at the level of epigenetics

28 and gene expression.

The epigenetic mark of DNA methylation has the potential to reflect past exposures with

long-lived marks on genes, while the transcriptome reflects current levels of gene expression in a

31 sampled tissue. To test the hypothesis that early-life wildfire smoke exposure would result in

32 detectable epigenetic differences to gene pathways reflecting cellular function, we performed the

33 integrated unbiased approaches of whole genome bisulfite sequencing (methylome) and RNA-

34 sequencing (transcriptome) from nasal epithelial samples collected the same cohorts of female

35 macaques examined a decade earlier for lung functions and immune responses. We identified a

36 large number of genes associated with early-life exposure-related differential methylation

37 involved in neuronal and immune signaling. In contrast, only one differentially expressed gene

38 (FLOT2) was stably associated with early-life wildfire smoke exposure.

\section{RESULTS}

41 Exposure to wildfire smoke during infancy is associated with long-lasting changes to DNA

42 methylation patterns in nasal epithelial cells.

43 To test the effects of early-life wildfire smoke-exposure on methylation status throughout the

44 genome, we performed whole genome bisulfite sequencing on nasal epithelial samples collected

45 from 22 adult rhesus macaques in 2019 ( 8 born in 2008 and exposed to high levels of $\mathrm{PM}_{2.5}$ and 
ozone due to wildfires, 14 born in 2009 and therefore has relatively low levels of exposure to $\mathrm{PM}_{2.5}$ and ozone; Figure 1, Table 1). Though there were several shared exposures to high levels of wildfire smoke $\mathrm{PM}_{2.5}\left(>35 \mathrm{ug} / \mathrm{m}^{3}\right.$, the 24-hour $\mathrm{PM}_{2.5}$ National Ambient Air Quality Standard) and ozone after the 2009 cohort was born (especially in the year of 2019), there was one high exposure event that only the 2008 cohort was exposed to in early-life (10 days above $35 \mathrm{ug} / \mathrm{m}^{3}$, Figure 1, Table 1). We assessed 26,609,677 CpG sites and identified 3370 differentially methylated regions (DMRs) between exposed and non-exposed samples (Figure 2, empirical p < 0.05 , differences in methylation $>5 \%$ ). The majority of these DMRs were hypermethylated in exposed animals $(2899, \sim 86 \%)$. A total of $114(3.38 \%)$ of these DMRs were primarily located in CpG islands (12), 287 (8.52\%) were located in CpG shores (0-2kb from island), 205 (6.08\%) were located in $\mathrm{CpG}$ shelves (2-4kb from island), and $2764(82.02 \%)$ were in the open sea $(>4 \mathrm{~kb}$ from island). This distribution was significantly different than expected by chance, with an enrichment towards $\mathrm{CpG}$ islands, shores, and shelves compared to regions assayed (Supplementary Figure 1). These 3370 DMRs were annotated to 2139 genes (Supplementary Table 1), of which 1852 genes were associated with DMRs hypermethylated in the exposed group, while 376 genes were associated with DMRs hypomethylated in the exposed group, and 89 genes were associated with both hypermethylated and hypomethylated DMRs (examples of DMRs shown in Figure 3). The DMRs were significantly more associated with promoters and exons than expected by chance, while they were less associated with intergenic regions than expected by chance (Supplementary Figure 2). The genes associated with DMRs as a whole were significantly enriched (FDR < 0.05) for 186 IPA canonical pathways, including axonal guidance signaling, synaptogenesis signaling pathway, protein kinase A signaling, IL-15 production, CXCR4 signaling, and Th1 and Th2 activation pathway (Figure 4, Supplementary Table 2). 
69 Genes associated with hypermethylated DMRs were enriched for 187 IPA pathways, 168 of which were also enriched in genes associated with DMRs as a whole. The 19 unique IPA

71 pathways enriched in hypermethylated DMRs include 14-3-3-mediated signaling, LPS-

72 stimulated MAPK Signaling, and $N F-\kappa B$ activation by viruses (Supplementary Table 3 ). Genes associated with hypomethylated DMRs were enriched for 41 IPA pathways, 23 of which were also enriched in genes associated with DMRs as a whole. The 18 unique IPA pathways enriched in hypomethylated DMRs include dermatan sulfate biosynthesis, xenobiotic metabolism PXR signaling pathway, and HOTAIR regulatory pathway (Supplementary Table 4).

\section{Impact of wildfire smoke-associated DNA methylation changes on TF binding.}

As the binding of transcription factors (TFs) are often influenced by DNA methylation, we performed a HOMER analysis to determine whether any transcription factor binding sites were enriched in these wildfire smoke-associated DMRs (13). A total of 131 transcription factor motifs were enriched in all DMRs $(q<0.05$; Supplementary Table 5). Eight of the top ten most highly enriched TF motifs are part of the bZIP TF family (shown in Table 2). When testing for TF binding site enrichment in only DMRs that were hypermethylated in exposed macaques, six of the top ten were part of the bZIP TF family, while none of the top ten enriched TF binding sites in hypomethylated DMRs were part of the bZIP TF family (five out of ten contained homeobox motifs). Interestingly, the TFs whose binding sites were most enriched in all wildfire smoke-associated DMRs were primarily unmethylated (Table 2) in other ChIP-seq datasets (14), so the differential methylation could theoretically have a large impact on transcription factor binding and expression (15). In support of this, DNA methylation generally inhibits binding of bZIP TF members to DNA $(15,16)$. 

types.

94 In order to understand the gene regulatory role of regions with wildfire smoke-DMRs, we

95 searched for the enrichment of 15 pre-defined chromatin states across 127 epigenomes from

97 maculatta coordinates into human (hg38) coordinates and using LOLA, the DMRs as a whole were enriched for bivalent chromatin marks (top odds ratio for any mark $=1.46$, q-value $<3 \mathrm{x}$ any mark $=2.05$, q-value $<0.02$; Figure 5B), though hypermethylated DMRs showed enrichment as well (Figure 5C).

To determine whether early-life exposure to wildfire smoke leads to detectable differences in gene expression later in life, we performed RNA-sequencing on 15 female rhesus macaques (6 born in 2008 and exposed to wildfire smoke, 9 born in 2009 and not directly exposed to the 2009

110 California wildfires). A principal component analysis (PCA) and hierarchical clustering of all

111 detected transcripts were performed to visualize how samples clustered based on expression

112 (Supplementary Figure 3D). The top two principal components in a principal component analysis 113 (PCA) explained 62\% of the variation in the dataset. Exposed and non-exposed samples did not 114 cluster separately in either the PCA or the hierarchical clustering analysis, implying no 
115 widespread transcriptomic difference between exposed and non-exposed individuals. After

116 multiple hypothesis correction (FDR $<0.05$, fold change $\geq 1.2$; Supplementary Table 6), there

117 was only one differentially expressed gene (FLOT2; Supplementary Table 6). None of the genes

118 annotated to DMRs were significantly differentially expressed.

119 To identify co-expressed genes whose expression correlated with wildfire smoke-

120 exposure status, we performed a weighted gene co-expression network analysis (WGCNA) (19).

121 We identified 16 co-expressed modules using WGCNA. None of the modules were significantly

122 associated with early-life exposure status $(\mathrm{p}<0.05)$. The module that best correlated with

123 exposure status was the purple module $(\mathrm{p}=0.1$; consisting of 585 genes, including IFI44,

124 IFNA21, and IL24; Supplementary Figure 4, Supplementary Table 7). No genes in this module

125 were significantly differentially expressed at an individual level, 19 genes were associated with

126 significant DMRs, and two genes had significantly correlated methylation and expression. The

127 genes in this module were enriched (FDR < 0.05) for 21 IPA pathways, including EIF2

128 signaling, mTOR signaling, Th17 activation pathway, and interferon signaling (Supplementary

129 Table 8).

131 Correlation of DNA methylation and gene expression differences resulting from wildfire smoke

132 exposure during infancy

133 Out of the 2139 genes associated with DMRs, 2128 had enough corresponding expression data to

134 evaluate the correlation between expression and methylation. To identify genes where

135 differential methylation may be ultimately leading to differential expression, we calculated the

136 spearman rank correlation between methylation and expression levels for genes that were

137 associated with DMRs. In total, 172 genes were significantly correlated (spearman p-value < 
138 0.05), with 76 genes showing a negative correlation and 96 showing a positive correlation

139 between methylation and expression (Supplementary Table 9, two examples are shown in Figure

140 6). These 172 genes were enriched for 32 IPA pathway terms, including leukocyte extravasation

141 signaling, CCR5 signaling in macrophages, and MIF regulation of innate immunity

142 (Supplementary Table 10).

\section{DISCUSSION}

145 Utilizing rhesus macaques that experienced the harsh conditions of the 2008 California wildfire

146 season in their first three months, we have elucidated some of the long-term effects of early-life

147 exposure to wildfire smoke. Baseline methylation profiles generally clustered better by exposure

148 status than expression profiles (Supplementary Figure 3). Many genes (2139) were associated

149 with differentially methylated regions between exposed and control macaques (empirical p <

150 0.05), while only 1 gene (FLOT2) was differentially expressed between these groups after

151 multiple hypothesis correction (FDR < 0.05). Out of the genes associated with differentially

152 methylated regions, 172 had methylation levels that significantly correlated with expression

153 levels across samples, indicating that the overall epigenetic regulatory landscape ultimately led

154 to few significant differences in baseline expression. However, the changes in DNA methylation

155 were significantly enriched at promoters and enhancers, and located at regions that transcription

156 factors may bind, suggesting that they may have an impact on gene regulation.

158 belongs to the lipid raft family. Flotillins are implicated in variety of cellular functions, including

159 regulation of G-protein coupled receptor signaling (20), endocytosis (21), cell-cell adhesion (22),

160 uropod formation and migratory capacity of neutrophils and monocytes (23) and T cells (24). 
161 FLOT2 also protected lung epithelial cells from Fas-signaling mediated apoptosis (25), and silica

162 nanoparticles were found in Flotillin-1 and -2 marked vesicles in alveolar epithelial cell (26).

163 However, its role in response to wildfire smoke exposure has not been reported. One potential

164 explanation for the few gene expression changes despite more widespread methylation

165 differences is that many of these DMRs are in regions associated with bivalent chromatin marks.

166 The differential methylation at these regions may not affect gene expression because the bivalent

167 chromatin marks generally keep expression repressed, but poised for rapid activation during

168 early development (27) or in cancer (28). This would imply that some of the methylation

169 differences were due to early-life events that were not reflected in baseline transcript levels later

170 in life. Additionally, although baseline gene expression was relatively similar between exposed

171 and control macaques, one hypothesis is that the altered regulatory landscape could lead to

172 differences in expression upon additional immune (or other) challenges. This hypothesis is

173 supported by a previous study on macaques from these same cohorts that found differences in

174 IL-6 (significant in males) and IL-8 (significant in females) production in peripheral blood

175 mononuclear cells (PBMC) from wildfire smoke-exposed macaques compared to controls after a

176 challenge with media, LPS, or flagellin (11). Out of 84 genes tested, only two (RELB and REL)

177 showed significant differences in expression following a media challenge (essentially a

178 comparison of baseline expression), while five genes were differentially expressed following a

179 challenge with either LPS or flagellin (11). RELB was the only gene that was differentially

180 expressed in all three tests, but the direction of change in challenged cells (increased RELB in

181 cells from exposed animals) was opposite of what was found at baseline (decreased RELB in

182 cells from exposed animals) (11). In summary, there were very few differences in baseline

183 expression in the previous study between exposed and control cells, and even when there was 
184

185

186

187

188

189

190

191

192

193

194

195

196

197

198

199

200

201

202

203

204

205

206

differential expression, those patterns changed or became non-significant following an immune challenge. While the sample types (PBMCs vs. nasal epithelium) and ages of the macaques (adolescents vs. adults) differ between the prior study and the current study, they both support that early exposure to wildfire smoke did not lead to drastic differences in baseline expression profiles between samples. Another potential explanation for differences in the degree of differential expression and differential methylation is that we had fewer samples for our differential expression analysis, potentially limiting our ability to identify differential expression compared to our ability to identify differential methylation. If there were widespread differences in expression due to exposure status, however, we expect that wildfire smoke-exposed samples would have clustered together in the principal component analysis and in hierarchical clustering analyses, so we postulate that this is not the major reason for the lack of differential expression.

\section{Long-term effects of wildfire smoke exposure on the methylome}

Our data implies that there are long-term effects on the methylome due to wildfire smoke exposures during infancy. DMRs were enriched for many pathways linked to asthma, COPD, or other pulmonary diseases, including IL-15 production $(29,30)$, CXCR4 signaling $(31,32)$, Actin cytoskeleton signaling $(33,34), \operatorname{VDR} / R X R$ activation $(35,36)$, Th1 and Th2 activation pathway

$(37,38)$, and Wnt/ $\beta$-catenin signaling $(39,40)$ (Supplementary Table 2$)$. Cytokines derived from T helper type 2 (Th2) cells have long been thought to play a critical role in allergic asthma through regulation of immunoglobulin $\mathrm{E}(\operatorname{IgE})$ synthesis $(38,41)$, but other $\mathrm{T}$ helper subsets (such as Th1) are starting to gain recognition for their role in asthma as well. Increased levels of the Th1 cytokine IFN- $\gamma$ have been shown to exacerbate existing asthmatic responses (42) and increase airway hyperresponsiveness (41) in transgenic mice. IFNGR2 (interferon gamma 
receptor 2) was differentially methylated in our comparison (as were several other Th1 related genes, including IL6R, LOC694631/IFNA1/13-like, and NFATC1), perhaps indicating that the early life wildfire smoke exposure has altered Th1 responses and resulted in differential responses to bacterial and viral infection. Additionally, hypermethylation of IL6 and IFNA13 was associated with idiopathic pulmonary fibrosis (IPF) (43), while hypermethylation of IL6R was associated with COPD in prior studies (44). IL6R and IFNA13 were also hypermethylated in exposed macaques in our current study (Supplementary Table 11), indicating that changes in the Th1 pathway may contribute to the reduction in lung function noted in macaques exposed to wildfire smoke early in life (11). Th2-related genes that were differentially methylated in our dataset include IL4R and TIMD4, while there were several genes associated with DMRs that were related to both the Th1 and Th2 pathways (including CD4, IL10, IL12RB2, NFATC2, RUNX3, and SOCS3). Hypermethylation of NFATC2 (44), RUNX3 (44), and SOCS3 (45) has been associated with COPD (Supplementary Table 11). These three genes were also hypermethylated in wildfire smoke-exposed macaques versus controls.

Deletion of Fra1, the transcription factor with the most enriched motif in the DMRs (Table 2, Supplementary Table 5), in mice led to greater levels of progressive interstitial fibrosis (46). Fra1 is a bZIP transcription factor and bZIP transcription factor binding is generally inhibited by methylation $(15,16)$. Meanwhile, overexpression of Fra2 (another highly enriched bZIP TF motif in the DMRs) in mice lead to non-allergic asthma development (47). The other bZIP transcription factors whose motifs were among the top ten enriched motifs have also all been linked to pulmonary disease (ATF3 (48), JunB (49), BATF (50), and AP-1 $(46,51)$ ). The role of bZIP transcription factors in pulmonary disease pathogenesis combined with the sensitivity of bZIP to changes in methylation imply that the differences in methylation noted 
230 between wildfire smoke-exposed and non-exposed macaques could greatly impact how bZIP

231 targets are regulated following a respiratory challenge. Overall, differences in methylation in Th1

232 and Th2-related genes (and the relation of those genes to asthma, IPF, and COPD pathogenesis;

233 see Supplementary Table 11) may explain the long-term differences in lung function previously

234 observed between wildfire smoke-exposed macaques and controls (11).

235 Interestingly, there is also recent research that suggests that exposure to air pollution can

236 have negative neuropsychological effects in children $(52,53)$. The DMRs from our dataset were

237 enriched in multiple IPA neurological pathways, including axonal guidance signaling (most

238 significant pathway), synaptogenesis signaling pathway (third most significant pathway), and

239 neuropathic pain signaling in dorsal horn neurons (Supplementary Table 2). Additionally, the

240 top enriched biological process term in GOfuncR (54) was neuron differentiation, while the top

241 enriched cellular component term was synapse (Supplementary Figure 5). The effect of wildfire

242 smoke on neurological development is understudied, but studies have shown that particles less

243 than $0.1 \mu \mathrm{m}$ in diameter (which are produced by wildfires) can cross the blood-brain barrier (55).

244 Additionally, exposure to these ultrafine particles has been associated with ADHD, autism, and

245 declines in school performance and memory in children (53). Along with this evidence from

246 prior studies, the differential methylation of regions near genes involved in neurological

247 pathways indicates that early-life wildfire smoke exposure could have a long-lasting impact on

248 nervous system function. 
252 In addition to directly studying genes and enriched pathways associated with DMRs, we also

253 wanted to identify genes that showed correlations between expression and methylation to get a

254 better understanding of how differences in methylation modify mRNA expression. Though only

255 one gene was differentially expressed between our groups following multiple hypothesis

256 correction (FLOT2), there were many more genes associated with DMRs that had a significant

257 correlation between methylation and expression (172 in total; Supplementary Table 9). MAPK10

258 (Spearman's $\rho \sim 0.75$ ) and WNT8B (Spearman's $\rho \sim 0.82$ ) were two other genes that were

259 associated with DMRs that showed a significant correlation between methylation and expression

260 (Supplementary Table 9). Wnt signaling has been linked to in utero lung development and

261 development/maturation during early life (alveologenesis) (56-58). Prior studies have shown that

$262 \mathrm{Wnt} / \beta$-catenin and the mitogen-activated protein kinase (MAPK) signaling pathway take part in

263 the airway remodeling process in asthma (39). In a mouse model of asthma, blocking Wnt

264 signaling reduced airway remodeling, while p38 MAPK expression was increased in asthmatic

265 mice compared to controls (39). MAPK10 expression was slightly higher on average in wildfire

266 smoke-exposed macaques than control macaques, and methylation was significantly positively

267 correlated with expression (hypermethylated in exposed animals). WNT8B expression was

268 slightly lower on average in exposed macaques, while methylation was significantly negatively

269 correlated with methylation (hypomethylated in exposed animals). Neither of these genes were

270 significantly differently expressed, however. Given the role of Wnt signaling and MAPK

271 signaling in airway remodeling, it seems possible that changes in gene regulation could have

272 contributed to the reduced lung function noted in wildfire smoke-exposed macaques (11). 
275 included only female rhesus macaques, but a prior study with these macaques noted significant

276 sex-specific differences in PBMCs challenged with LPS or flagellin. Male wildfire smoke-

277 exposed macaques had significantly higher levels of IL-8 compared to controls, while female

278 wildfire smoke-exposed macaques had significantly higher levels of IL-6 compared to controls

279 (11). While IL-6 was not differentially expressed or methylated in our exposed macaques

280 compared to controls, this does underscore that we may have missed some sex-specific

281 differences in gene expression or methylation by sampling only female macaques for our current

282 study. Indeed, studies have shown that there are sex-specific differences in expression between

283 female and male asthmatics $(59,60)$, implying that the molecular underpinnings of asthma and

284 other pulmonary issues may differ between the sexes. Additionally, our cohort of wildfire

285 smoke-exposed macaques was roughly one year older than our cohort of control macaques.

286 Studies have indicated that methylation patterns are associated with aging (epigenetic clocks) in

287 humans $(61,62)$, so this is likely the case for rhesus macaques as well. Out of 2139 genes that

288 were associated with DMRs in our dataset, 20 were differentially methylated in a pattern that

289 was consistent with the models from the previously referenced studies on epigenetic clocks.

290 Based on these results, most of the differential methylation we observed cannot be explained by

291 known differences in how methylation correlates with age. Another potential alternative

292 explanation is that the differences in methylation we observed were due to greater cumulative

293 exposure to pollutants in the older macaques. Table 1 shows that the difference in cumulative

294 exposures to high levels of $\mathrm{PM}_{2.5}$ and ozone between the two groups were roughly equivalent to

295 the differences observed in the first three months of life, implying that these early exposures

296 were key drivers of the noted differences between the groups. However, cumulative exposures 
297 below the current U.S. EPA standards were associated with increased mortality in a Medicare population (63), and they may also have an impact on the epigenome. The epigenetic effects of acute and chronic wildfire smoke exposure are worthy of further investigation. As previously discussed, we had a smaller sample size for our expression dataset $(n=13$ after removing two outliers) than our methylation dataset $(n=22)$. This could explain why we saw fewer changes in expression overall, however samples appeared to cluster more closely based on exposure status for the methylation dataset than the expression dataset (Supplementary Figure 3). The p-values

304 from DMRichR were empirical p-values calculated from permutation tests $(64,65)$. Although this puts our study at a higher risk of false-positive findings, these permutation p-values calculated by DMRichR were used to determine DMR significance in multiple published studies in combination with effect size (64-67). Given that our analysis of chromatin states relied on human hg38 annotations, we compared our macaque rheMac10 annotations to the hg38 annotations to make sure they were similar enough. About $67 \%$ of the DMR gene annotations were exact matches after lifting over the coordinates to hg 38 . While $30 \%$ of DMRs had a

311 different annotation, some of these differences were just due to differences in gene naming 312 convention between the species. For example, one DMR was annotated to LOC694631 313 (IFNA1/13-like) in rheMac10, while the lifted over DMR was annotated to IFNA13 in hg38. In a 314 broader pathway analysis, 90\% of the IPA pathways enriched in DMRs using rheMac10 315 annotations were also enriched when we used hg38 annotations. We also focused our discussion 316 on genes that were consistent between the two annotations. 
years (68)), the methylation profiles still clustered based on exposure status (Figure 2,

321 Supplementary Figure 3). This implies that there are long-term impacts of wildfire smoke

322 exposure on methylation, and that at least some of these changes are highly stable. An early

323 study on DNA methylation stability involved sampling individuals three days apart to check for

324 differences in DNA methylation. This study on 12 gene promoters indicated that methylation

325 stability was marker dependent and varied based on sequence composition (69). Meanwhile, a

326 large-scale study on how storage conditions affect methylation stability indicated that storing

327 DNA samples in temperatures as high as four degrees Celsius for up to 20 years had no

328 significant impact on methylation (70).

CONCLUSIONS:

331 In summary, our study revealed differences in methylation and gene expression in nasal

332 epithelial samples between macaques that were exposed to wildfire smoke during early life and

333 macaques that were not exposed to wildfire smoke during early life. The wildfire smoke

334 associated DMRs were enriched for a variety of immune processes, but there were few

335 significant expression differences at baseline between exposed and non-exposed macaques.

336 Given the differences in methylation, perhaps differences in expression between these two

337 groups would become apparent following an immune/respiratory challenge, but future studies

338 would be required to explore this hypothesis. Our study indicates that wildfire smoke exposure in

339 early life can have long-term impacts on the epigenome.

\section{METHODS:}


Animals

343 Wildfire smoke-exposed rhesus macaque monkeys born between April 1 and June 8, 2008 were

344 housed in outdoor facilities at the CNPRC from birth to now (Table 1). Monkeys born between

345 April 1 and June 8, 2009 were used as controls. PM2.5 and ozone were measured by a California

346 Air Resources Board air monitoring station (site no. 57,577) located 2.7 miles southeast of the

347 California National Primate Research Center on the University of California Davis campus

348 (Figure 1). Care and housing of animals complied with the provisions of the Institute of

349 Laboratory Animal Resources and conformed to practices established by the American

350 Association for Accreditation of Laboratory Animal Care. Procedures in this study were

351 approved by the UC Davis Institutional Animal Care and Use Committee.

352

Sample Collection and DNA/RNA Extraction

354 Nasal epithelium samples were collected from 22 female rhesus macaques (Macaca mulatta)

355 housed at the California National Primate Research Center. Exposures of these animals to

356 wildfire smoke were previously estimated (11). Eight of these macaques were born in 2008 and

357 exposed to wildfire smoke from birth to 3 months old, while the other 14 were born in 2009 with

358 low wildfire exposure from birth to 3 months old (Table 1, demographic comparison of two

359 groups). We collected these nasal epithelium samples in 2019. RNA and DNA were isolated

360 using the Allprep DNA and RNA kit (Qiagen) according to the manufacturer's instructions. 
363 Whole genome bisulfite sequencing (WGBS) libraries were prepared for all 22 samples. Library

364 quality was checked prior to sequencing using an Agilent 2100 Bioanalyzer system; library

365 concentration was measured using a Qubit DNA high sensitivity assay. Each library was

366 comprised of sample from a single individual; these individually barcoded libraries were then

367 pooled and sequenced on two lanes from a NovaSeq 6000 S4 flow cell at PE150 using Swift's

368 Accel-NGS Methyl-Seq Kit at the DNA Technologies and Expression Analysis Cores at the UC

369 Davis Genome Center. We sequenced approximately 475 million paired end reads per sample

370 that passed initial filters. Reads were demultiplexed using the bcl2fastq Illumina software.

WGBS read alignment, differential methylation analysis, pathway analysis, and chromatin state

373 analysis

374 The CpG_Me pipeline (71-74) was utilized to align the WGBS data. Reads were trimmed using

375 Trim Galore (73) to address methylation biases at the 5' and 3' end of reads (10 bases were

376 trimmed from the 3' end of both read 1 and read 2, and 10 and 20 bases were trimmed from the

3775 ' end of reads 1 and 2 respectively). The reads were aligned to the $M$. mulatta genome using

378 Bismark (72), which was also used to deduplicate the aligned reads and generate CpG count

379 matrices. Read quality and mapping quality were assessed using MultiQC (74). Differentially

380 methylated regions between exposed and non-exposed macaques were identified using

381 DMRichR $(64,75,76)$, which uses the dmrseq (75) and bsseq (76) algorithms. Animal weight

382 was adjusted for as a covariate. We used the default paramters for DMRichR, including requiring

383 at least $1 \mathrm{x}$ coverage for all samples for a $\mathrm{CpG}$, requiring a minimum of $5 \mathrm{CpGs}$ for a DMR,

384 performing 10 permutations for DMR and block analyses, and setting the single CpG coefficient

385 required to discover testable background regions to be at least 0.05 . Using DMRichR, candidate 
regions are identified based on differences in mean methylation between groups, then regionlevel metrics that account for mean methylation, $\mathrm{CpG}$ correlation, and coverage are computed.

These region-level metrics are then compared to a pooled null distribution generated via permutations to calculate an empirical $p$ value for each candidate region $(64,65)$. Bsseq $(76)$ was used to generate individual smoothed methylation values and heatmap visualizations. IPA (QIAGEN Inc., https://www.qiagenbioinformatics.com/products/ingenuitypathway-analysis) was used for pathway enrichment analysis. We also used GOfuncR (54) for GO enrichments based on DMR coordinates rather than gene names. HOMER (13) was used to identify enriched transcription factor binding motifs in the DMRs $(\mathrm{p}<0.05)$, while we utilized MethMotif $(14)$ to characterize methylation frequency of transcription factors whose binding motifs were enriched in the DMRs. We used the UCSC liftover tool (77) to lift DMR coordinates from rheMac10 to hg38 because chromatin state information was not available for M. mulatta. Locus Overlap Analysis (LOLA) (78) was used to determine whether DMRs were enriched for chromHMM (79) states relative to the background regions. The spearman correlation coefficient between gene expression and methylation levels for genes associated with DMRs was used to determine whether significant methylation changes were associated with changes in gene expression $(\mathrm{p}<$ $0.05)$.

\section{Library preparation for RNA-seq}

RNAseq libraries were prepared for a total of 15 samples: 6 from wildfire smoke-exposed individuals and 9 from non-exposed individuals (Supplementary Table 12, comparison of these two groups). As some of the RNA samples were of low quantity, a special low-input RNA-seq pipeline were applied at the Genomics, Epigenomics and Sequencing Core of University of 
409 Cincinnati $(80,81)$. Briefly, polyA RNA was isolated using NEBNext Poly(A) mRNA Magnetic

410 Isolation Module (New England BioLabs, Ipswich, MA) and enriched using SMARTer Apollo

411 NGS library prep system (Takara Bio USA, Mountain View, CA). Libraries were prepared using

412 NEBNext Ultra II Directional RNA Library Prep Kit (New England BioLabs), indexed, pooled

413 and sequenced using Nextseq 550 sequencer (Illumina, San Diego, CA). Approximately 40

414 million reads passing filter per sample were generated under the sequencing setting of single read

4151 1x85 bp. Reads were demultiplexed and adapters were trimmed using the bcl2fastq Illumina

416 software.

RNA-seq read alignment, differential expression analysis, pathway analysis, and co-expression

418 analysis

Read quality was checked using FastQC (82), then the reads were aligned to the Macaca mulatta genome (rheMac10, GenBank assembly accession: GCA_003339765.3) with Bowtie2 (83).

421 Transcripts were quantified using RSEM (84). The data from $R S E M$ was congregated and

422 converted into DESeq2 (85) format using tximport (86). Sample clustering by expression

423 (investigated via principal component analysis and hierarchical clustering) and detection of

424 differentially expressed genes between wildfire smoke-exposed and non-exposed samples was

425 done using DESeq2 (85). Individual weight was included as a covariate in the differential

426 expression analysis. Two samples (one wildfire smoke-exposed and one non-exposed sample)

427 were excluded from all subsequent RNA-sequencing analyses because they were identified as

428 outliers in the hierarchical clustering analysis (Supplementary Figure 6). The resulting log-fold

429 change values were shrunken (following the recommendation from the DESeq2 reference

430 manual) using apeglm (87). Differentially expressed genes had FDR $<0.05$ and an absolute

431 shrunken fold change of at least 1.2. The Ingenuity Pathway Analysis (IPA) software (QIAGEN 
432 Inc., https://www.qiagenbioinformatics.com/products/ingenuitypathway-analysis) was used for

433 pathway analysis. Significantly enriched pathways in IPA had a p-value $<0.05$.

434 Co-expressed modules of genes were found using WGCNA (19). The soft threshold (power) was

435 set to 8 based on a plot of soft threshold vs scale free topology model fit. Modules that were too

436 similar to one another (below a height of 0.5 ) were merged into one module. After merging, the

437 final co-expression modules were tested for significant associations with wildfire smoke

438 exposure and animal weight. Pathways enriched in genes in modules of interest were identified

439 using IPA.

440 DECLARATIONS

441 Ethics approval and consent to participate

442 Procedures in this study were approved by the UC Davis Institutional Animal Care and Use

443 Committee.

444 Consent for publication

445 Not applicable.

$446 \quad$ Availability of data and materials

447 WGBS and RNA-seq data will be deposited to GEO upon manuscript acceptance.

448 Competing interests

449 The authors declare they have no competing interests.

$450 \quad$ Funding

451 This study was supported by a pilot grant from Environmental Health Sciences Center (NIEHS-

452 P30 ES006096) awarded to HJ. HJ was also supported by an Environmental Health Sciences

453 Scholar Award (P30 ES006096), and NIH/NIAID R01AI141569-1A1. BL was supported 
454 Canadian Institutes of Health Research (CIHR) postdoctoral fellowship (MFE-146824) and a

455 CIHR Banting postdoctoral fellowship (BPF-162684).

\section{Authors' contributions}

457 HJ conceived the study in discussion with LAM and JML. APB drafted the manuscript with the

458 help of HJ, BIL, JML and LAM. LC extracted DNA and RNA from nasal samples, prepared

459 WGBS libraries. APB performed WGBS analysis with the help of BIL. APB performed other

460 data analysis and visualization in discussion with HJ. All authors have approved the final

461 version of this manuscript.

462 Acknowledgements

463 Nasal sampling was performed by staff members at CNPRC Research Services. The sequencing

464 was carried out at the DNA Technologies and Expression Analysis Cores at the UC Davis

465 Genome Center, supported by NIH Shared Instrumentation Grant 1S10OD010786-01. 
467 Table 1: Demographic characteristics of animal populations

Participants

$\mathrm{N}$

Age at sample collection (yr)

Weight at sample collection $(\mathrm{kg})$

Genetic background

Indian

Mixed Indian-Chinese

Corral diversity

Maternal Background

Age at parturition (yr)

Genetic background

Indian

Mixed Indian-Chinese

Corral diversity

Ambient pollutants months 0-3

Days with $\mathrm{PM}_{2.5}$ higher than $35 \mathrm{ug} / \mathrm{m}^{3}$

Mean $\mathrm{PM}_{2.5}$ concentration $\left(\mu \mathrm{g} / \mathrm{m}^{3}\right)$

Median $\mathrm{PM}_{2.5}$ concentration $\left(\mu \mathrm{g} / \mathrm{m}^{3}\right)$

Hours over California 1-h ozone standard

Mean ozone level (ppm)

Median ozone level (ppm)

Cumulative exposures through sampling date
8
$11.2 \pm 0.2$

$8.61 \pm 1.60$

7

13

1.0

1

8

13

1.0
7

1

8
12

2

14

1.0

1.0
$10 \pm 1.0$

$0 \pm 0$

$\mathrm{P}<0.0001$

$14.6 \pm 16.3$

$9.1 \pm 3.7$

$\mathrm{P}<0.0001$

$9.5(6.0-15.6)$

$8.8(6.4-11.4)$

$\mathrm{P}<0.0001$

$13 \pm 2.9$

$0 \pm 0$

$\mathrm{P}<0.0001$

$0.032 \pm 0.020$

$0.029 \pm 0.017$

$\mathrm{P}<0.001$

$0.030(0.016-0.045)$

$0.027(0.016-0.040)$

$\mathrm{P}<0.001$ 
Days with $\mathrm{PM}_{2.5}$ higher than $35 \mathrm{ug} / \mathrm{m}^{3}$

Mean $\mathrm{PM}_{2.5}$ concentration $\left(\mu \mathrm{g} / \mathrm{m}^{3}\right)$

Median $\mathrm{PM}_{2.5}$ concentration $\left(\mu \mathrm{g} / \mathrm{m}^{3}\right)$

Hours over California 1-h ozone standard

Mean ozone level (ppm)

Median ozone level (ppm)
$40 \pm 0$

$9.3 \pm 8.0$

$8.4(5.5-13.4)$

$24 \pm 0$

$0.026 \pm 0.015$

$0.025(0.015-0.036)$
$29 \pm 0.4$

$\mathrm{P}<0.0001$

$9.1 \pm 7.7$

$\mathrm{P}<0.0001$

$8.6(6.3-11.4)$

$\mathrm{P}<0.0001$

$7 \pm 0$

N/A (0 SD)

$\mathrm{P}<0.001$

$\mathrm{P}<0.001$

Note: Age, weight and exposures are shown as mean $\pm(\mathrm{SD})$ or median (interquartile range), and compared using $\mathrm{t}$ test. Categorical variables (genetic background and corral diversity) are reported as group-specific numerical frequency and compared using Fisher's exact tests. $35 \mathrm{ug} / \mathrm{m}^{3}$ is the 24-hour $\mathrm{PM}_{2.5}$ National Ambient Air Quality Standard. 0.09ppm is the California 1-h ozone standard. 


\section{Figure legends}

470

471

472

473

474

475

476

477

478

479

480

481

482

483

484

485

486

487

Figure 1: Average daily $\mathrm{PM}_{2.5}$ from April 2008 through October 2019 at the California Air Resources Board air monitoring station (site no. 57,577) located 2.7 miles southeast of the California National Primate Research Center on the University of California Davis campus. The dotted line at $35 \mathrm{ug} / \mathrm{m}^{3}$ represent the 24-hour $\mathrm{PM}_{2.5}$ National Ambient Air Quality Standard. Note the arrow pointing to the early-life exposure event in macaques born in 2008. All other exposure events were shared between the two groups.

Figure 2: Heatmap showing sample clustering based on methylation. The heatmap includes only differentially methylated regions (DMRs). The heatmap was normalized on a per row basis for visualization, therefore the values on the scales are relative rather than absolute.

Figure 3: Examples of differentially methylated regions (DMRs) between rhesus macaques exposed in the first three months of life to wildfire smoke and those that were not. A) IL4R. B) RXRG. C) TLR5. D) ITGB6. Each dot represents the methylation percentage of one individual at one $\mathrm{CpG}$ site, while each line represents the smoothed average methylation level moving across the region. The red shaded boxes denote the specific DMR locations. Tracks for CpG islands (if present) or genes are included underneath each plot. For the gene tracks, a solid box indicates an exon, while the arrows indicate the direction of transcription.

Figure 4: Enriched pathway analyses for differentially methylated regions (DMRs). Only the top ten (out of 186) enriched Ingenuity Pathway Analysis (IPA) canonical pathways are shown. 
$491 \quad$ Figure 5: Enrichment in chromHMM (79) states in A) all differentially methylated regions

492 (DMRs), B) DMRs that were hypomethylated in wildfire smoke-exposed macaques, and C)

493 DMRs that were hypermethylated in wildfire smoke-exposed macaques. The rows in the plot

494 represent different datasets from different cell types from the NIH Roadmap Epigenomics

495 Consortium (88). Epithelial and IMR90 are highlighted in the plots, as these are the closest to the

496 nasal epithelial samples in our current study.

498 Figure 6. Correlation plots between expression and methylation for A) MAPK10and B) CD44.

499 Each individual point represents one sample. Expression and methylation were significantly

500 correlated (spearman p-value $<0.05$ ) for both genes. 


\section{References}

1. Knowlton K. Where there's fire, there's smoke: wildfire smoke affects communities distant from deadly flames. 2013.

2. Dockery DW. Health effects of particulate air pollution. Ann Epidemiol. 2009;19(4):25763.

3. Brunekreef B, Holgate ST. Air pollution and health. Lancet. 2002;360(9341):1233-42. 4. Li YJ, Takizawa H, Kawada T. Role of oxidative stresses induced by diesel exhaust particles in airway inflammation, allergy and asthma: their potential as a target of chemoprevention. Inflamm Allergy Drug Targets. 2010;9(4):300-5.

5. Zhou Z, Liu Y, Duan F, Qin M, Wu F, Sheng W, et al. Transcriptomic Analyses of the Biological Effects of Airborne PM2.5 Exposure on Human Bronchial Epithelial Cells. PloS one. 2015;10(9):e0138267-e.

6. Gehring U, Gruzieva O, Agius RM, Beelen R, Custovic A, Cyrys J, et al. Air pollution exposure and lung function in children: the ESCAPE project. Environ Health Perspect. 2013;121(11-12):1357-64.

7. Urman R, McConnell R, Islam T, Avol EL, Lurmann FW, Vora H, et al. Associations of children's lung function with ambient air pollution: joint effects of regional and near-roadway pollutants. Thorax. 2014;69(6):540-7.

8. Spears D, Dey S, Chowdhury S, Scovronick N, Vyas S, Apte J. The association of earlylife exposure to ambient PM2.5 and later-childhood height-for-age in India: an observational study. Environmental Health. 2019;18(1):62.

9. Rosa MJ, Hair GM, Just AC, Kloog I, Svensson K, Pizano-Zárate ML, et al. Identifying critical windows of prenatal particulate matter (PM(2.5)) exposure and early childhood blood pressure. Environ Res. 2020;182:109073.

10. To T, Zhu J, Stieb D, Gray N, Fong I, Pinault L, et al. Early life exposure to air pollution and incidence of childhood asthma, allergic rhinitis and eczema. European Respiratory Journal. 2020;55(2):1900913.

11. Black C, Gerriets JE, Fontaine JH, Harper RW, Kenyon NJ, Tablin F, et al. Early Life Wildfire Smoke Exposure Is Associated with Immune Dysregulation and Lung Function Decrements in Adolescence. Am J Respir Cell Mol Biol. 2017;56(5):657-66.

12. Jones PA. Functions of DNA methylation: islands, start sites, gene bodies and beyond. Nature reviews Genetics. 2012;13(7):484-92.

13. Heinz S, Benner C, Spann N, Bertolino E, Lin YC, Laslo P, et al. Simple combinations of lineage-determining transcription factors prime cis-regulatory elements required for macrophage and B cell identities. Mol Cell. 2010;38(4):576-89.

14. Xuan Lin QX, Sian S, An O, Thieffry D, Jha S, Benoukraf T. MethMotif: an integrative cell specific database of transcription factor binding motifs coupled with DNA methylation profiles. Nucleic Acids Research. 2018;47(D1):D145-D54.

15. Yin Y, Morgunova E, Jolma A, Kaasinen E, Sahu B, Khund-Sayeed S, et al. Impact of cytosine methylation on DNA binding specificities of human transcription factors. Science. 2017;356(6337).

16. Héberlé É, Bardet AF. Sensitivity of transcription factors to DNA methylation. Essays in biochemistry. 2019;63(6):727-41.

17. Ernst J, Kellis M. ChromHMM: automating chromatin-state discovery and characterization. Nature methods. 2012;9(3):215-6. 
18. Bernstein BE, Mikkelsen TS, Xie X, Kamal M, Huebert DJ, Cuff J, et al. A bivalent chromatin structure marks key developmental genes in embryonic stem cells. Cell. 2006;125(2):315-26.
19. Langfelder P, Horvath S. WGCNA: an R package for weighted correlation network analysis. BMC Bioinformatics. 2008;9(1):559.

20. Sugawara Y, Nishii H, Takahashi T, Yamauchi J, Mizuno N, Tago K, et al. The lipid raft proteins flotillins/reggies interact with Galphaq and are involved in Gq-mediated p38 mitogenactivated protein kinase activation through tyrosine kinase. Cell Signal. 2007;19(6):1301-8. 21. Otto GP, Nichols BJ. The roles of flotillin microdomains--endocytosis and beyond. Journal of cell science. 2011;124(Pt 23):3933-40.

22. Bodin S, Planchon D, Rios Morris E, Comunale F, Gauthier-Rouviere C. Flotillins in intercellular adhesion - from cellular physiology to human diseases. Journal of cell science. 2014;127(Pt 24):5139-47.

23. Ludwig A, Otto GP, Riento K, Hams E, Fallon PG, Nichols BJ. Flotillin microdomains interact with the cortical cytoskeleton to control uropod formation and neutrophil recruitment. The Journal of cell biology. 2010;191(4):771-81. 24. Giri B, Dixit VD, Ghosh MC, Collins GD, Khan IU, Madara K, et al. CXCL12-induced partitioning of flotillin-1 with lipid rafts plays a role in CXCR4 function. European journal of immunology. 2007;37(8):2104-16.

25. Wei S, Moon HG, Zheng Y, Liang X, An CH, Jin Y. Flotillin-2 modulates fas signaling mediated apoptosis after hyperoxia in lung epithelial cells. PloS one. 2013;8(10):e77519.

\section{Kasper J, Hermanns MI, Bantz C, Utech S, Koshkina O, Maskos M, et al. Flotillin-} involved uptake of silica nanoparticles and responses of an alveolar-capillary barrier in vitro. Eur J Pharm Biopharm. 2013;84(2):275-87.

27. Mikkelsen TS, Ku M, Jaffe DB, Issac B, Lieberman E, Giannoukos G, et al. Genomewide maps of chromatin state in pluripotent and lineage-committed cells. Nature. 2007;448(7153):553-60.

28. Bernhart SH, Kretzmer H, Holdt LM, Juhling F, Ammerpohl O, Bergmann AK, et al. Changes of bivalent chromatin coincide with increased expression of developmental genes in cancer. Sci Rep. 2016;6:37393.

29. Jonakowski M, Zioło J, Koćwin M, Przemęcka M, Mokros Ł, Panek M, et al. Role of IL15 in the modulation of TGF- $\beta 1$-mediated inflammation in asthma. Exp Ther Med. 2017;14(5):4533-40.

30. Liu Z, Fan W, Chen J, Liang Z, Guan L. The role of Interleukin 15 in protein degradation in skeletal muscles in rats of chronic obstructive pulmonary disease. Int J Clin Exp Med. 2015;8(2):1976-84.

31. Chen H, Xu X, Teng J, Cheng S, Bunjhoo H, Cao Y, et al. CXCR4 inhibitor attenuates allergen-induced lung inflammation by down-regulating MMP-9 and ERK1/2. Int J Clin Exp Pathol. 2015;8(6):6700-7.

32. Henrot P, Prevel R, Berger P, Dupin I. Chemokines in COPD: From Implication to Therapeutic Use. International journal of molecular sciences. 2019;20(11):2785. 33. Tang DD, Gerlach BD. The roles and regulation of the actin cytoskeleton, intermediate filaments and microtubules in smooth muscle cell migration. Respiratory Research. 2017;18(1):54. 
34. D'Anna C, Cigna D, Di Sano C, Di Vincenzo S, Dino P, Ferraro M, et al. Exposure to cigarette smoke extract and lipopolysaccharide modifies cytoskeleton organization in bronchial epithelial cells. Experimental Lung Research. 2017;43(9-10):347-58.

35. Iqbal SF, Freishtat RJ. Mechanism of action of vitamin D in the asthmatic lung. J Investig Med. 2011;59(8):1200-2.

36. Hu G, Dong T, Wang S, Jing H, Chen J. Vitamin D(3)-vitamin D receptor axis suppresses pulmonary emphysema by maintaining alveolar macrophage homeostasis and function. EBioMedicine. 2019;45:563-77.

37. Martín-Orozco E, Norte-Muñoz M, Martínez-García J. Regulatory T Cells in Allergy and Asthma. Frontiers in Pediatrics. 2017;5(117).

38. Barnes PJ. Th2 cytokines and asthma: an introduction. Respiratory research. 2001;2(2):64-5.

39. Jia X-X, Zhu T-T, Huang Y, Zeng X-X, Zhang H, Zhang W-X. Wnt/ $\beta$-catenin signaling pathway regulates asthma airway remodeling by influencing the expression of c-Myc and cyclin D1 via the p38 MAPK-dependent pathway. Experimental and therapeutic medicine. 2019;18(5):3431-8.

40. Guo L, Wang T, Wu Y, Yuan Z, Dong J, Li Xo, et al. WNT/ $\beta$-catenin signaling regulates cigarette smoke-induced airway inflammation via the PPAR $\delta / \mathrm{p} 38$ pathway. Laboratory Investigation. 2016;96(2):218-29.

41. Kim Y-M, Kim Y-S, Jeon SG, Kim Y-K. Immunopathogenesis of allergic asthma: more than the th2 hypothesis. Allergy Asthma Immunol Res. 2013;5(4):189-96.

42. Durrant DM, Metzger DW. Emerging roles of T helper subsets in the pathogenesis of asthma. Immunol Invest. 2010;39(4-5):526-49.

43. Sanders YY, Ambalavanan N, Halloran B, Zhang X, Liu H, Crossman DK, et al. Altered DNA methylation profile in idiopathic pulmonary fibrosis. Am J Respir Crit Care Med. 2012;186(6):525-35.

44. Morrow JD, Cho MH, Hersh CP, Pinto-Plata V, Celli B, Marchetti N, et al. DNA methylation profiling in human lung tissue identifies genes associated with COPD. Epigenetics. 2016;11(10):730-9.

45. Vucic EA, Chari R, Thu KL, Wilson IM, Cotton AM, Kennett JY, et al. DNA methylation is globally disrupted and associated with expression changes in chronic obstructive pulmonary disease small airways. Am J Respir Cell Mol Biol. 2014;50(5):912-22.

46. Rajasekaran S, Vaz M, Reddy SP. Fra-1/AP-1 Transcription Factor Negatively Regulates Pulmonary Fibrosis In Vivo. PLOS ONE. 2012;7(7):e41611.

47. Gungl A, Biasin V, Wilhelm J, Olschewski A, Kwapiszewska G, Marsh LM. Fra2 Overexpression in Mice Leads to Non-allergic Asthma Development in an IL-13 Dependent Manner. Front Immunol. 2018;9(2018).

48. Gilchrist M, Henderson WR, Jr., Clark AE, Simmons RM, Ye X, Smith KD, et al. Activating transcription factor 3 is a negative regulator of allergic pulmonary inflammation. $\mathrm{J}$ Exp Med. 2008;205(10):2349-57.

49. Chen S, Yun F, Yao Y, Cao M, Zhang Y, Wang J, et al. USP38 critically promotes asthmatic pathogenesis by stabilizing JunB protein. Journal of Experimental Medicine. 2018;215(11):2850-67.

50. Übel C, Sopel N, Graser A, Hildner K, Reinhardt C, Zimmermann T, et al. The activating protein 1 transcription factor basic leucine zipper transcription factor, ATF-like (BATF), 
672

673

674

675

676

677

678

679

regulates lymphocyte- and mast cell-driven immune responses in the setting of allergic asthma. $\mathrm{J}$ Allergy Clin Immunol. 2014;133(1):198-206.e1-9.

51. Caramori G, Casolari P, Adcock I. Role of transcription factors in the pathogenesis of asthma and COPD. Cell Commun Adhes. 2013;20(1-2):21-40.

52. Suades-González E, Gascon M, Guxens M, Sunyer J. Air Pollution and Neuropsychological Development: A Review of the Latest Evidence. Endocrinology. 2015;156(10):3473-82.

53. Holm SM, Miller MD, Balmes JR. Health effects of wildfire smoke in children and public health tools: a narrative review. Journal of Exposure Science \& Environmental Epidemiology. 2020.

54. Grote S. GOfuncR: Gene ontology enrichment using FUNC. R package version 1.10.0 ed2020.

55. Schraufnagel DE. The health effects of ultrafine particles. Exp Mol Med. 2020;52(3):311-7.

56. Frank DB, Peng T, Zepp JA, Snitow M, Vincent TL, Penkala IJ, et al. Emergence of a Wave of Wnt Signaling that Regulates Lung Alveologenesis by Controlling Epithelial SelfRenewal and Differentiation. Cell Reports. 2016;17(9):2312-25.

57. Hussain M, Xu C, Lu M, Wu X, Tang L, Wu X. Wnt/ $\beta$-catenin signaling links embryonic lung development and asthmatic airway remodeling. Biochimica et Biophysica Acta (BBA) Molecular Basis of Disease. 2017;1863(12):3226-42.

58. De Langhe SP, Reynolds SD. Wnt signaling in lung organogenesis. Organogenesis. 2008;4(2):100-8.

59. Gautam Y, Afanador Y, Abebe T, López JE, Mersha TB. Genome-wide analysis revealed sex-specific gene expression in asthmatics. Hum Mol Genet. 2019;28(15):2600-14.

60. Mersha TB, Martin LJ, Biagini Myers JM, Kovacic MB, He H, Lindsey M, et al. Genomic architecture of asthma differs by sex. Genomics. 2015;106(1):15-22.

61. Hannum G, Guinney J, Zhao L, Zhang L, Hughes G, Sadda S, et al. Genome-wide Methylation Profiles Reveal Quantitative Views of Human Aging Rates. Molecular Cell. 2013;49(2):359-67.

62. Horvath S. DNA methylation age of human tissues and cell types. Genome biology. 2013;14(10):R115.

63. Shi L, Zanobetti A, Kloog I, Coull BA, Koutrakis P, Melly SJ, et al. Low-Concentration PM2.5 and Mortality: Estimating Acute and Chronic Effects in a Population-Based Study. Environ Health Perspect. 2016;124(1):46-52.

64. Laufer BI, Hwang H, Vogel Ciernia A, Mordaunt CE, LaSalle JM. Whole genome bisulfite sequencing of Down syndrome brain reveals regional DNA hypermethylation and novel disorder insights. Epigenetics. 2019;14(7):672-84.

65. Mordaunt CE, Jianu JM, Laufer BI, Zhu Y, Hwang H, Dunaway KW, et al. Cord blood DNA methylome in newborns later diagnosed with autism spectrum disorder reflects early dysregulation of neurodevelopmental and X-linked genes. Genome Med. 2020;12(1):88. 66. Murat El Houdigui S, Adam-Guillermin C, Armant O. Ionising Radiation Induces Promoter DNA Hypomethylation and Perturbs Transcriptional Activity of Genes Involved in Morphogenesis during Gastrulation in Zebrafish. International Journal of Molecular Sciences. 2020;21(11):4014. 
67. Laufer BI, Gomez JA, Jianu JM, LaSalle JM. Stable DNMT3L overexpression in SHSY5Y neurons recreates a facet of the genome-wide Down syndrome DNA methylation signature. Epigenetics \& Chromatin. 2021;14(1):13.

68. Colman RJ, Anderson RM, Johnson SC, Kastman EK, Kosmatka KJ, Beasley TM, et al. Caloric restriction delays disease onset and mortality in rhesus monkeys. Science. 2009;325(5937):201-4.

69. Byun H-M, Nordio F, Coull BA, Tarantini L, Hou L, Bonzini M, et al. Temporal Stability of Epigenetic Markers: Sequence Characteristics and Predictors of Short-Term DNA Methylation Variations. PLOS ONE. 2012;7(6):e39220.

70. Li Y, Pan X, Roberts ML, Liu P, Kotchen TA, Jr AWC, et al. Stability of global methylation profiles of whole blood and extracted DNA under different storage durations and conditions. Epigenomics. 2018;10(6):797-811.

71. Laufer BI, Hwang H, Jianu JM, Mordaunt CE, Korf IF, Hertz-Picciotto I, et al. Low-pass whole genome bisulfite sequencing of neonatal dried blood spots identifies a role for RUNX1 in Down syndrome DNA methylation profiles. Human Molecular Genetics. 2020;29(21):3465-76. 72. Krueger F, Andrews SR. Bismark: a flexible aligner and methylation caller for BisulfiteSeq applications. Bioinformatics (Oxford, England). 2011;27(11):1571-2.

73. Martin M. Cutadapt removes adapter sequences from high-throughput sequencing reads. 2011. 2011;17(1):3.

74. Ewels P, Magnusson M, Lundin S, Käller M. MultiQC: summarize analysis results for multiple tools and samples in a single report. Bioinformatics. 2016;32(19):3047-8.

75. Korthauer K, Chakraborty S, Benjamini Y, Irizarry RA. Detection and accurate false discovery rate control of differentially methylated regions from whole genome bisulfite sequencing. Biostatistics. 2018;20(3):367-83.

76. Hansen KD, Langmead B, Irizarry RA. BSmooth: from whole genome bisulfite sequencing reads to differentially methylated regions. Genome Biology. 2012;13(10):R83.

77. Kuhn RM, Haussler D, Kent WJ. The UCSC genome browser and associated tools. Brief Bioinform. 2013;14(2):144-61.

78. Sheffield NC, Bock C. LOLA: enrichment analysis for genomic region sets and regulatory elements in $\mathrm{R}$ and Bioconductor. Bioinformatics. 2015;32(4):587-9.

79. Ernst J, Kellis M. Chromatin-state discovery and genome annotation with ChromHMM. Nat Protoc. 2017;12(12):2478-92.

80. Walsh KB, Zhang X, Zhu X, Wohleb E, Woo D, Lu L, et al. Intracerebral Hemorrhage Induces Inflammatory Gene Expression in Peripheral Blood: Global Transcriptional Profiling in Intracerebral Hemorrhage Patients. DNA Cell Biol. 2019;38(7):660-9.

81. Rapp SJ, Dershem V, Zhang X, Schutte SC, Chariker ME. Varying Negative Pressure Wound Therapy Acute Effects on Human Split-Thickness Autografts. J Burn Care Res. 2020;41(1):104-12.

82. Andrews S. FastQC: A Quality Control Tool for High Throughput Sequence Data. 2010. 83. Langmead B, Salzberg SL. Fast gapped-read alignment with Bowtie 2. Nature Methods. 2012;9(4):357-9.

84. Li B, Dewey CN. RSEM: accurate transcript quantification from RNA-Seq data with or without a reference genome. BMC Bioinformatics. 2011;12(1):323.

85. Love MI, Huber W, Anders S. Moderated estimation of fold change and dispersion for RNA-seq data with DESeq2. Genome Biology. 2014;15(12):550. 
725 86. Soneson C, Love M, Robinson M. Differential analyses for RNA-seq: transcript-level

726 estimates improve gene-level inferences [version 1; peer review: 2 approved]. F1000Research.

$727 \quad 2015 ; 4(1521)$.

728 87. Zhu A, Ibrahim JG, Love MI. Heavy-tailed prior distributions for sequence count data:

729 removing the noise and preserving large differences. Bioinformatics. 2018;35(12):2084-92.

730 88. Kundaje A, Meuleman W, Ernst J, Bilenky M, Yen A, Heravi-Moussavi A, et al.

731 Integrative analysis of 111 reference human epigenomes. Nature. 2015;518(7539):317-30. 
Average Daily $\mathrm{PM}_{2.5}$ Over Time, Davis, CA

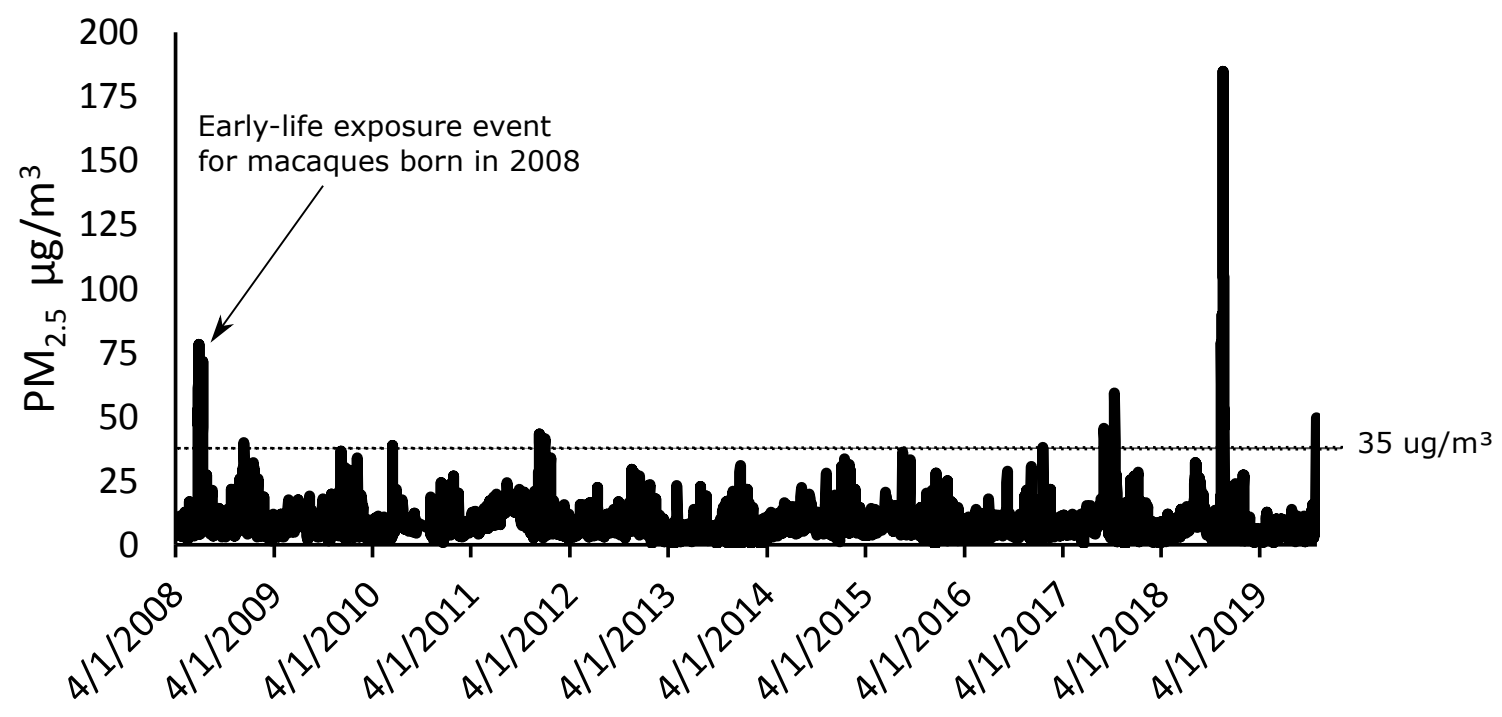

Figure 1 


\section{Clustering by methylation, differentially methylated regions only}

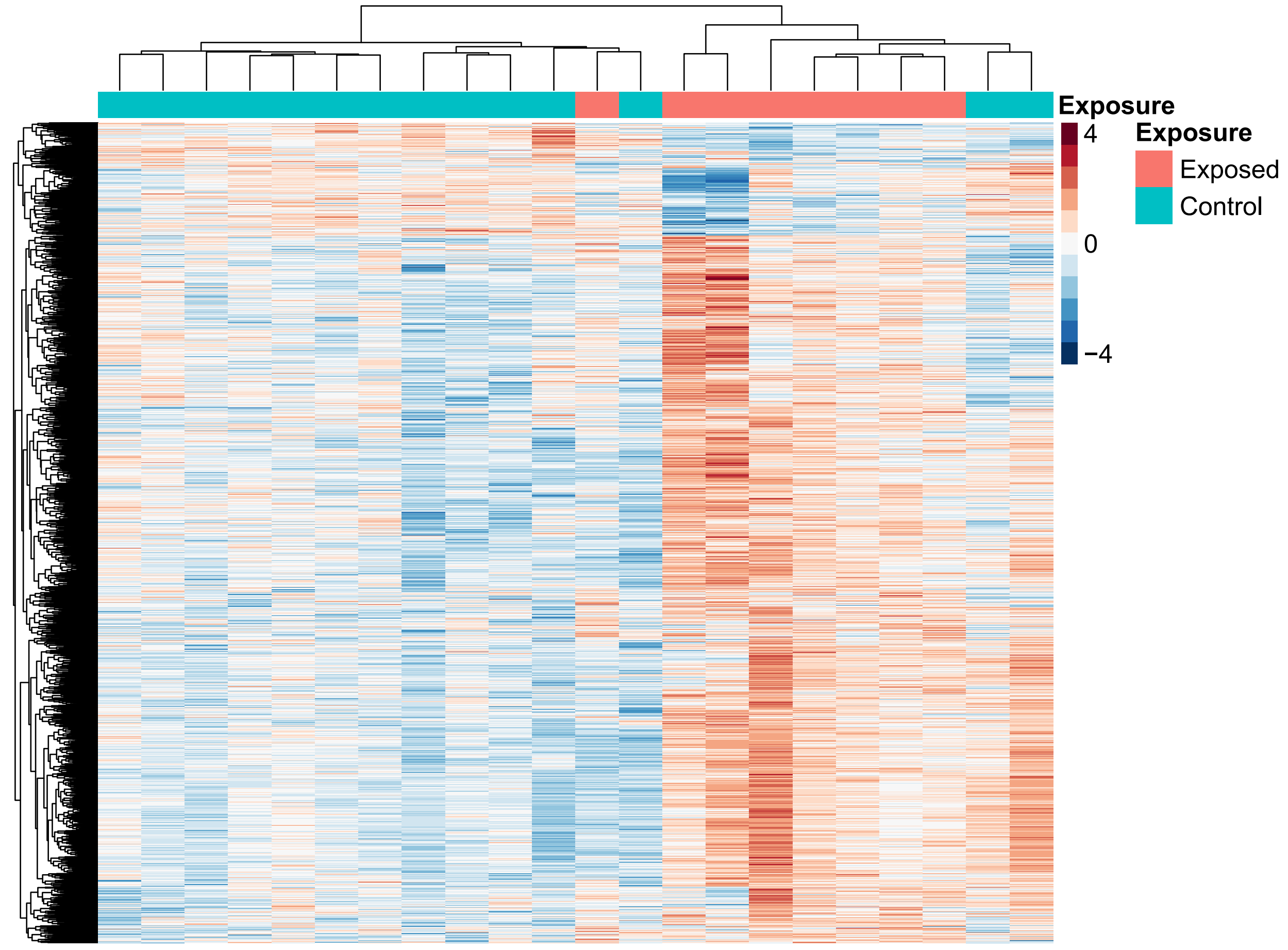

Figure 2 
A

IL4R methylation

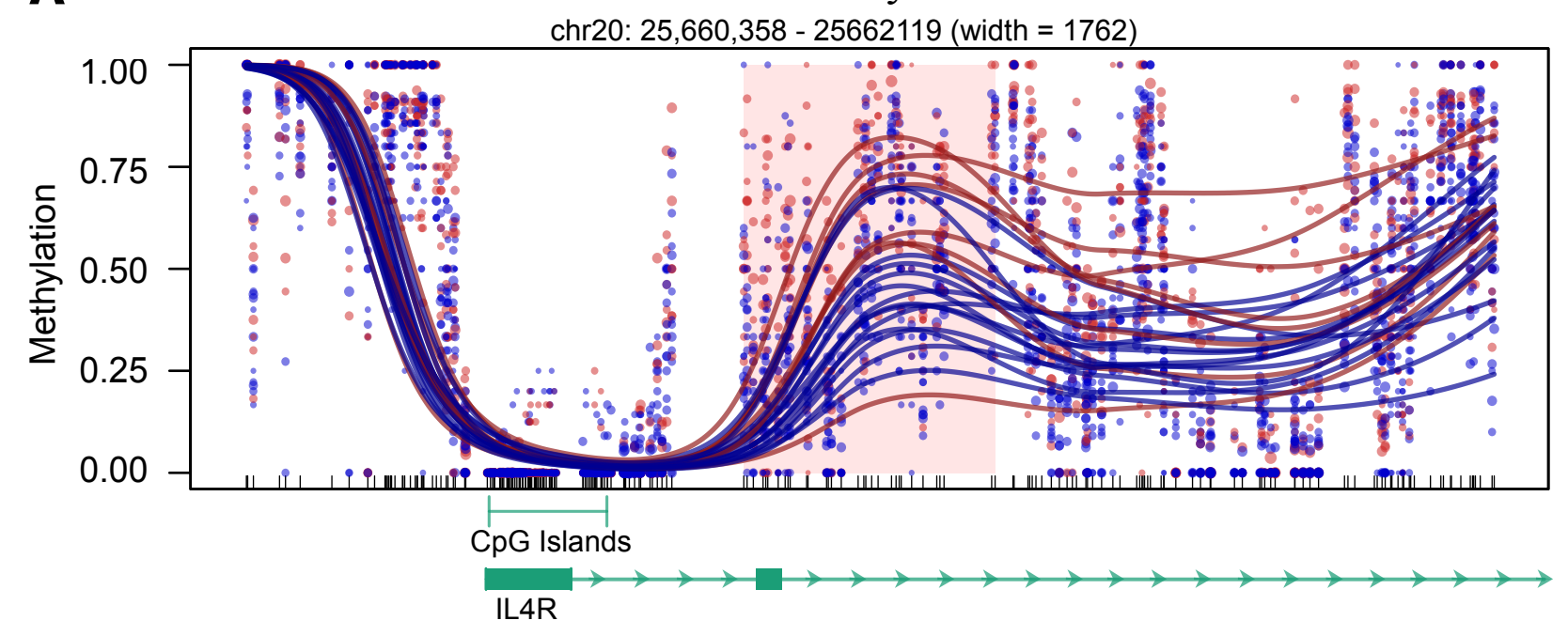

TLR5 methylation

C

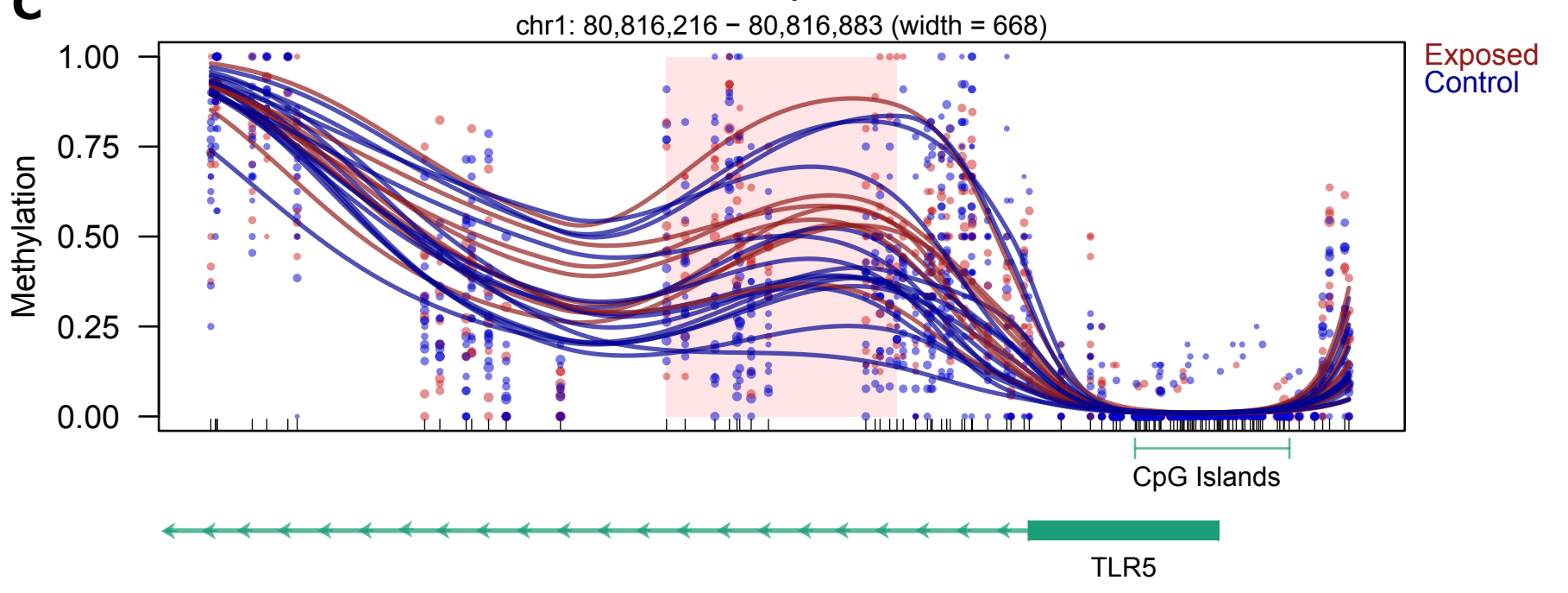

Exposed
B

RXRG methylation

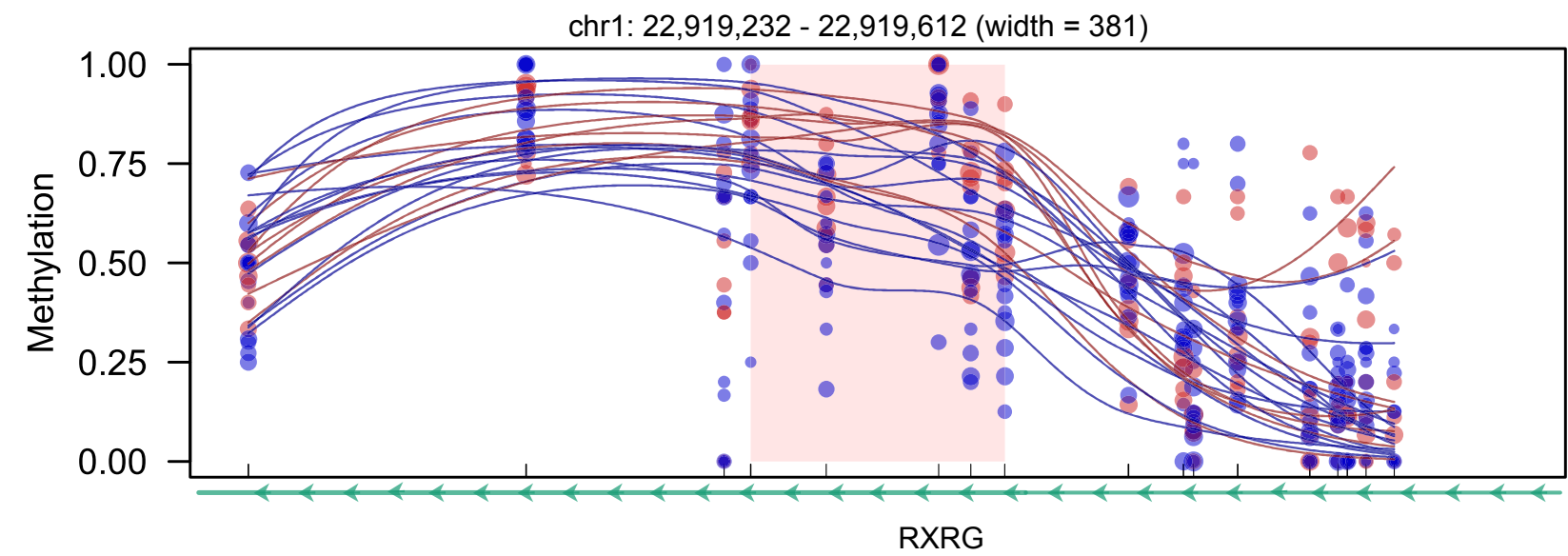

Exposed
Control

D

ITGB6 methylation

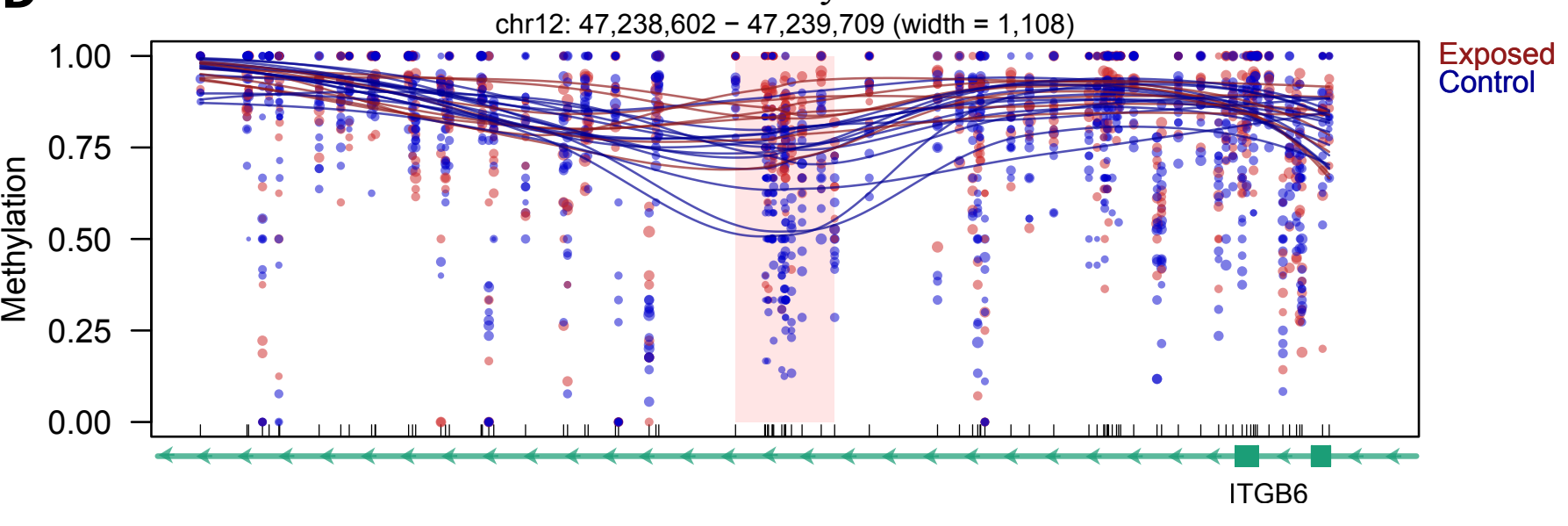

Figure 3 
Top 10 enriched canonical pathways in differentially methylated regions -log(p-value)

Axonal Guidance Signaling

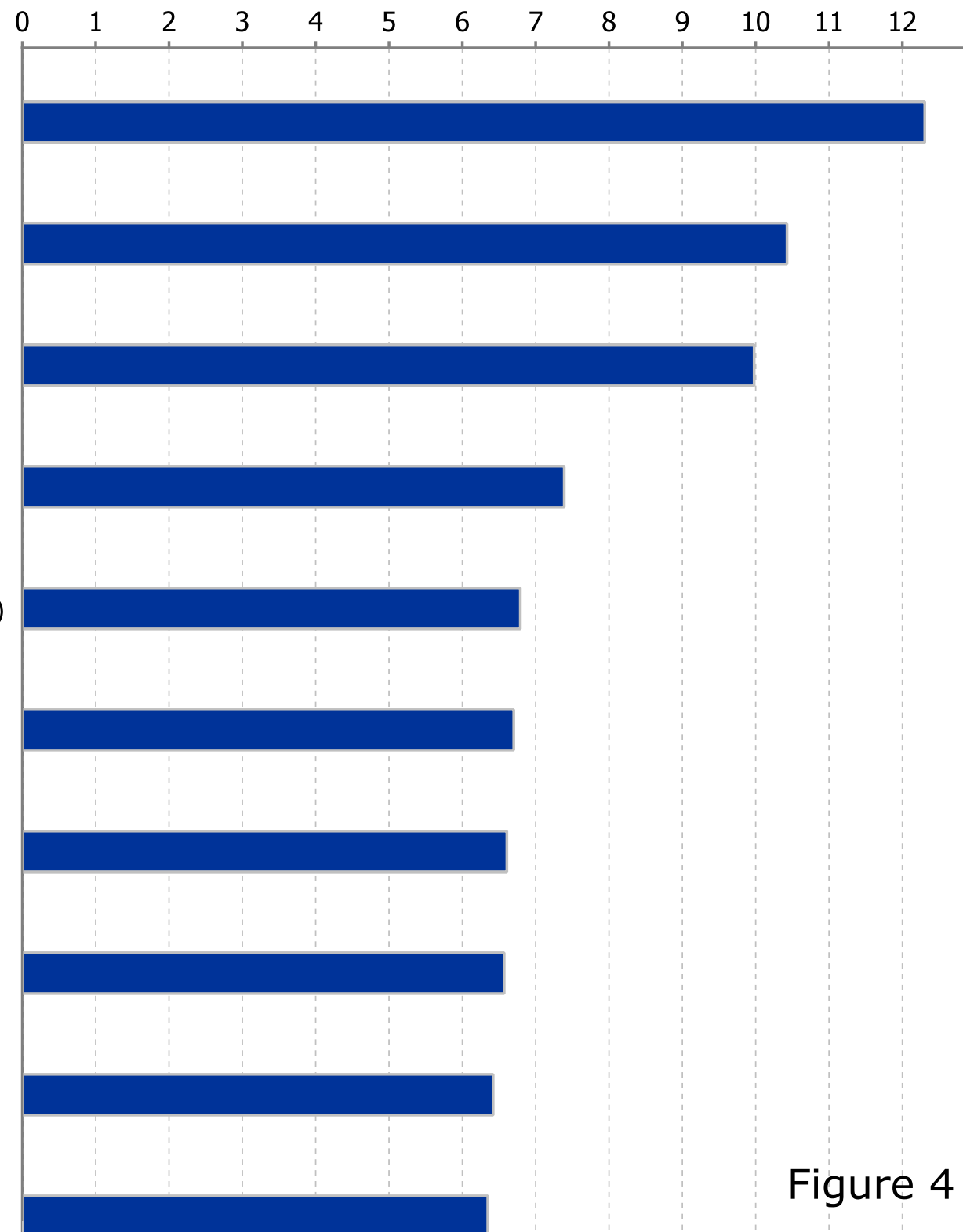

RhoGDI Signaling

Synaptogenesis Signaling Pathway

Protein Kinase A Signaling

Cardiac Hypertrophy Signaling (Enhanced)

Molecular Mechanisms of Cancer

CREB Signaling in Neurons

Signaling by Rho Family GTPases

Ephrin Receptor Signaling 


\section{A}

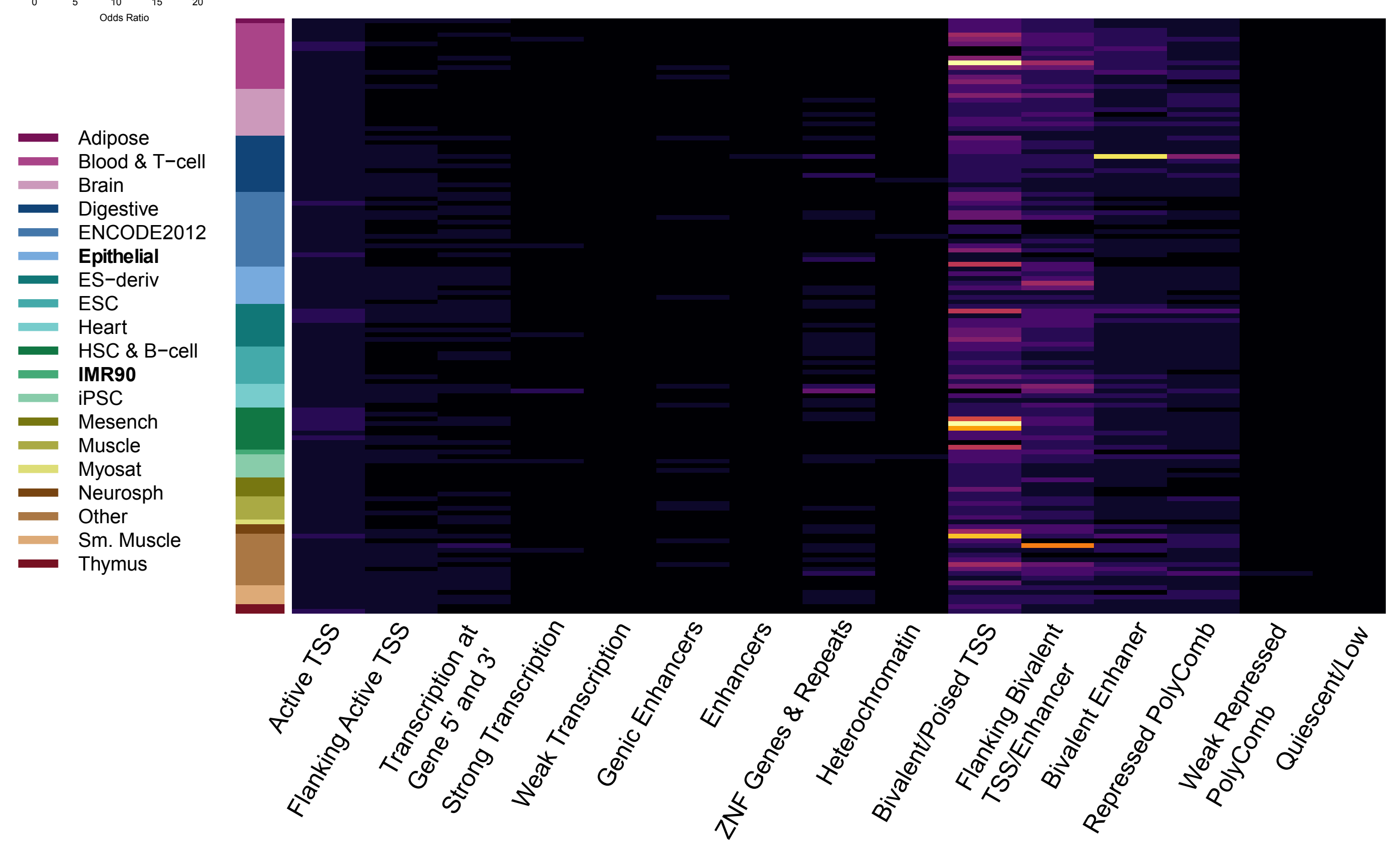

B
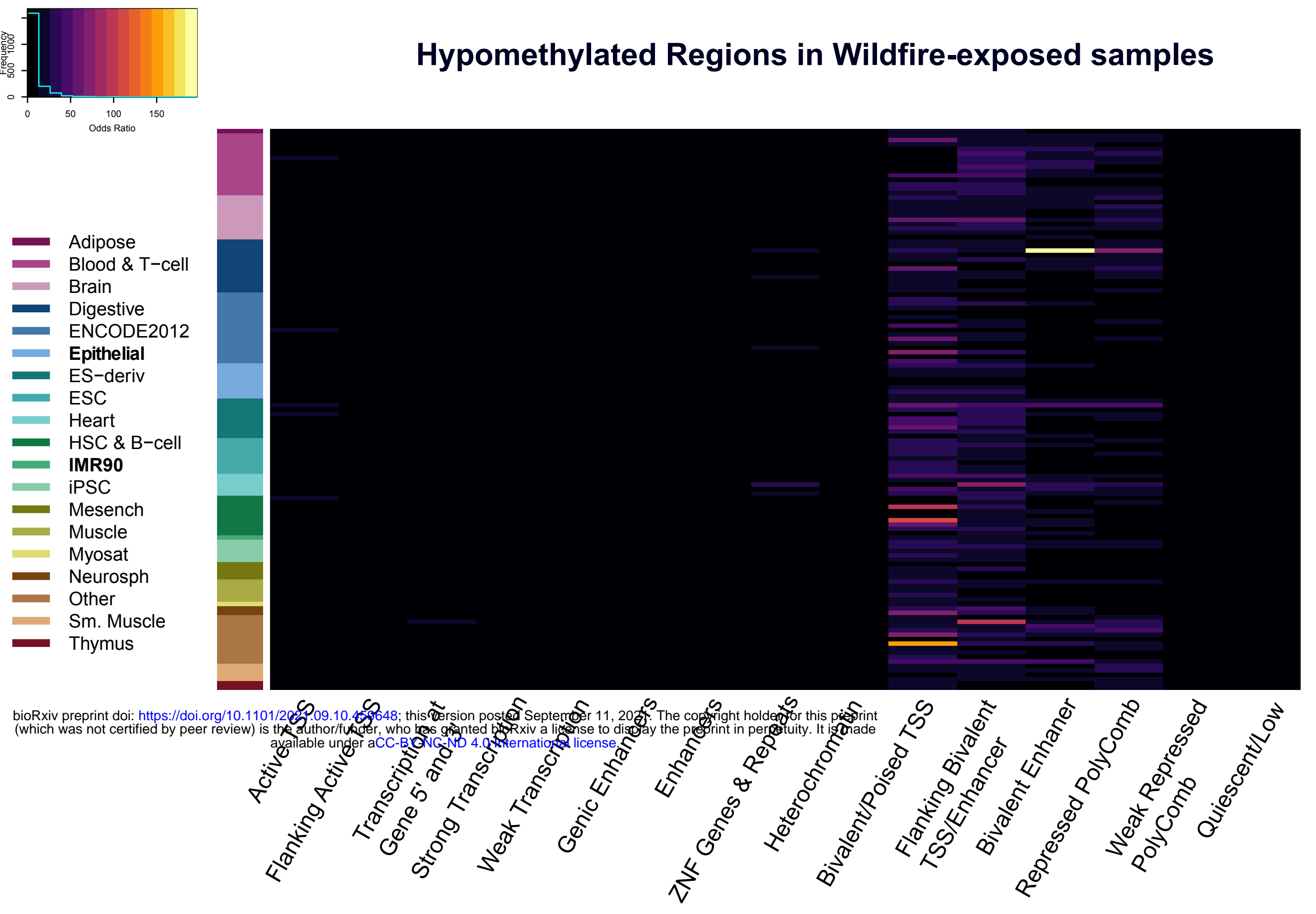

C

Hypermethylated Regions in Wildfire-exposed Samples

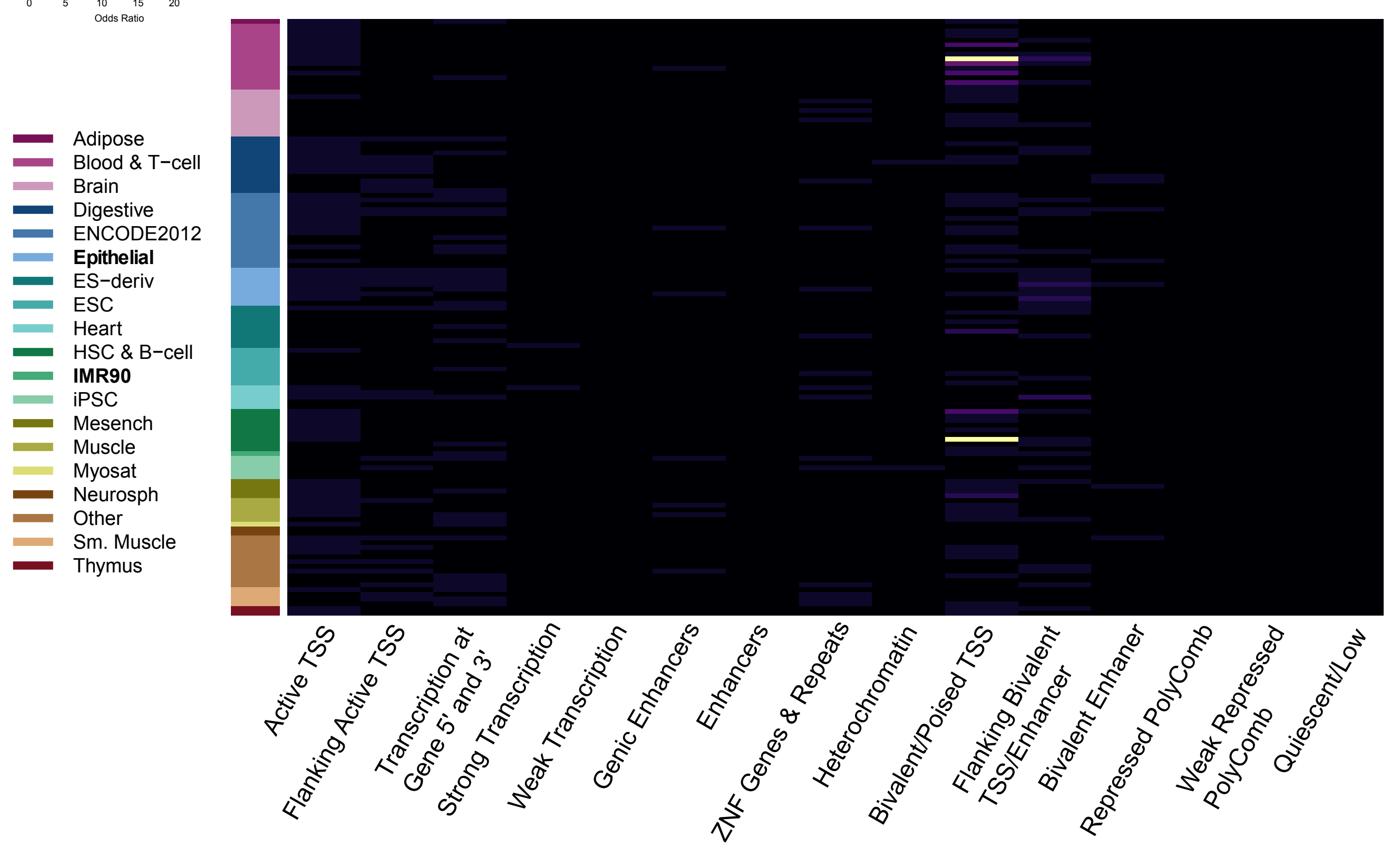




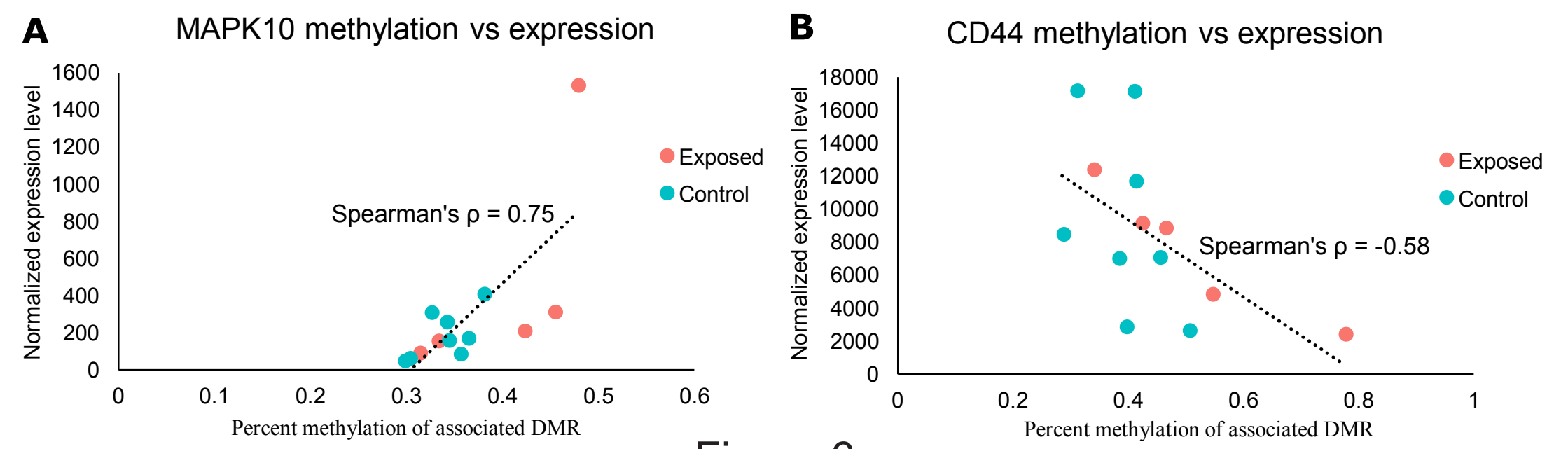

Figure 6 


\begin{tabular}{cccc}
\hline Motif & unmethylated & partially methylated & methylated \\
\hline Fra1/FOSL1 & 0.68 & 0.32 & 0.00 \\
Atf3 & 0.82 & 0.18 & 0.01 \\
JunB & 0.88 & 0.12 & 0.00 \\
BATF & 0.69 & 0.28 & 0.03 \\
Fra2/FOSL2 & 0.90 & 0.10 & 0.00 \\
AP-1/Jun & 0.81 & 0.19 & 0.00 \\
p63/TP53 & 0.46 & 0.35 & 0.19 \\
NF1-halfsite(CTF)/NFIA & 0.64 & 0.31 & 0.05 \\
\hline
\end{tabular}

\section{Table 2}


Supplementary Table 1. Differentially methylated regions between rhesus macaques exposed to wildfire smoke in early life and rhesus macaques with no early life exposure to wildfire smoke. Note: betaCoefficient is presented with respect to exposed macaques (i.e. a positive betaCoefficient implies hypermethylation in exposed macaques compared to control macaques)

Supplementary Table 2. Canonical pathways from Ingenuity Pathway Analysis that were enriched in all differentially methylated regions.

Supplementary Table 3. Canonical pathways from Ingenuity Pathway Analysis that were enriched in differentially methylated regions hypermethylated in macaques exposed to wildfire smoke in early life.

Supplementary Table 4. Canonical pathways from Ingenuity Pathway Analysis that were enriched in differentially methylated regions hypomethylated in macaques exposed to wildfire smoke in early life.

Supplementary Table 5. Transcription factor binding site motifs that were significantly enriched in differentially methylated regions (from HOMER (1)).

Supplementary Table 6. The differentially expressed gene between rhesus macaques exposed to wildfire smoke in early life and rhesus macaques with no early life exposure to wildfire smoke. Note: $\log 2$ FoldChange is presented with respect to exposed macaques (i.e. a positive 
log2FoldChange implies greater expression in exposed macaques compared to control macaques).

Supplementary Table 7. Genes in the purple module (the module most significantly associated with exposure) from the weighted gene coexpression network analysis (WGCNA (2)).

Supplementary Table 8. Canonical pathways from Ingenuity Pathway Analysis that were enriched in genes in the purple module from the WGCNA (2) analysis.

Supplementary Table 9. Genes that showed significant correlation $(p \leq 0.05)$ between methylation and expression across all samples.

Supplementary Table 10. Canonical pathways from Ingenuity Pathway Analysis that were enriched in genes that had significantly correlated methylation and expression.

Supplementary Table 11. Comparison between differentially methylated genes from the current study and other studies on respiratory diseases.

Supplementary Table 12. Extended information on the samples in our current study.

Supplementary Figure 1. Enrichment of different $\mathrm{CpG}$ features associated with all differentially methylated regions, regions hypermethylated in wildfire-exposed macaques, and regions 
hypomethylated in wildfire-exposed macaques. Asterisks indicate a significant deficit or enrichment of the feature in a given set $(\mathrm{p} \leq 0.05)$.

Supplementary Figure 2. Enrichment of different genic features associated with all differentially methylated regions, regions hypermethylated in wildfire-exposed macaques, and regions hypomethylated in wildfire-exposed macaques. Asterisks indicate a significant deficit or enrichment of the feature in a given set $(\mathrm{p} \leq 0.05)$.

Supplementary Figure 3. Heatmaps showing sample clustering by A) methylation and B) gene expression, and principal component analysis showing sample clustering by C) methylation and D) gene expression.

Supplementary Figure 4. Module-trait relationship between clusters identified in WGCNA and either exposure or animal weight. The top number in each box is the correlation value (ranging from -1 to 1), while the bottom number in parentheses is the p-value for this correlation.

Supplementary Figure 5. Top enriched biological process, cellular component, and molecular function gene ontology terms identified by GOfuncR (3) associated with differentially methylated regions between wildfire-exposed macaques and control macaques.

Supplementary Figure 6. Heatmap showing all samples clustering by gene expression. Gene expression data from the two leftmost samples were removed from the study as outliers. 


\section{References}

1. Heinz S, Benner C, Spann N, Bertolino E, Lin YC, Laslo P, et al. Simple combinations of lineage-determining transcription factors prime cis-regulatory elements required for macrophage and $\mathrm{B}$ cell identities. Mol Cell. 2010;38(4):576-89.

2. Langfelder P, Horvath S. WGCNA: an R package for weighted correlation network analysis. BMC Bioinformatics. 2008;9(1):559.

3. Grote S. GOfuncR: Gene ontology enrichment using FUNC. R package version 1.10.0 ed2020. 


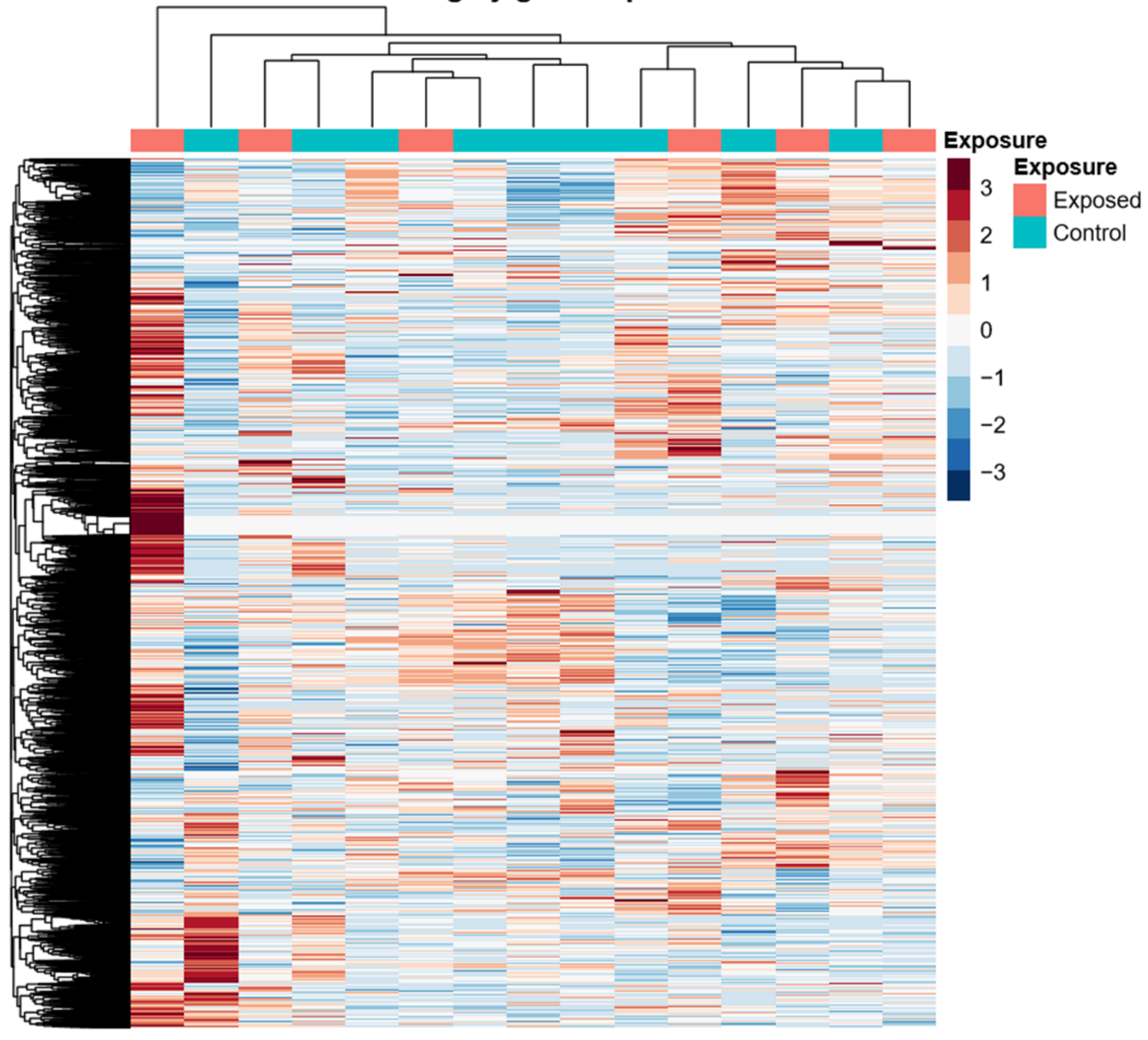

Supplemntary Figure 1 


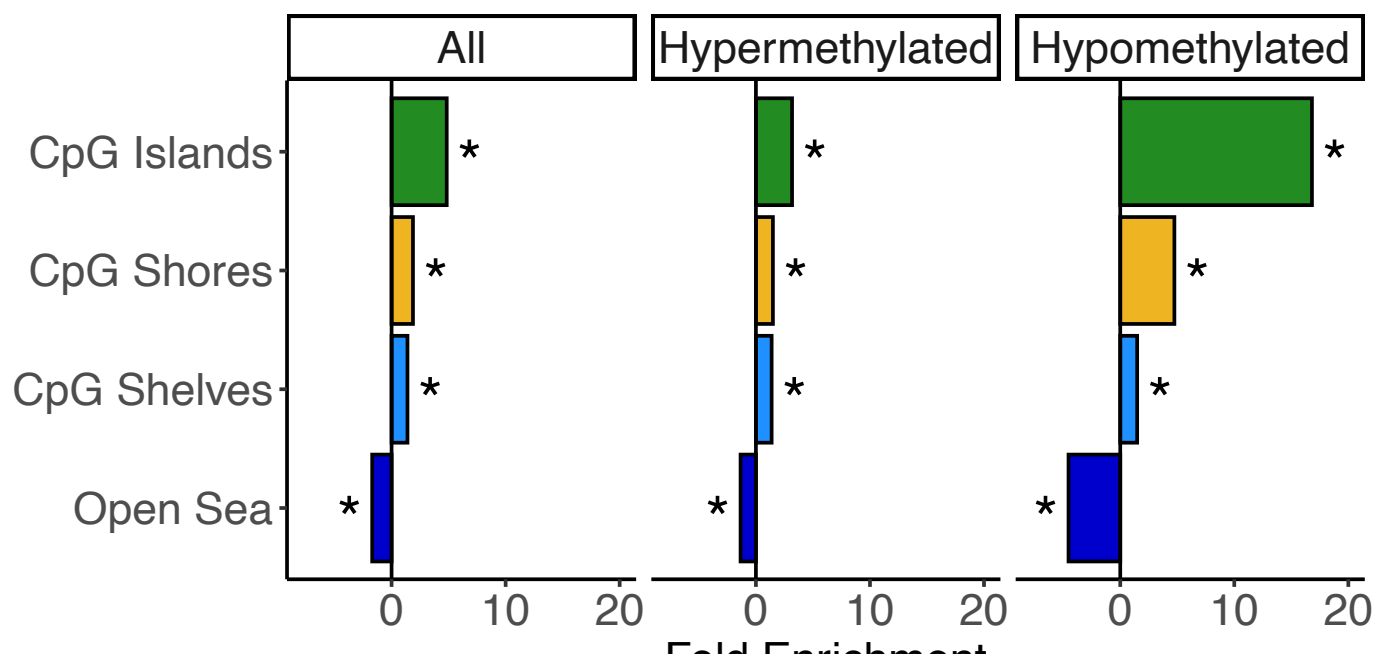

Fold Enrichment

Supplemntary Figure 2 


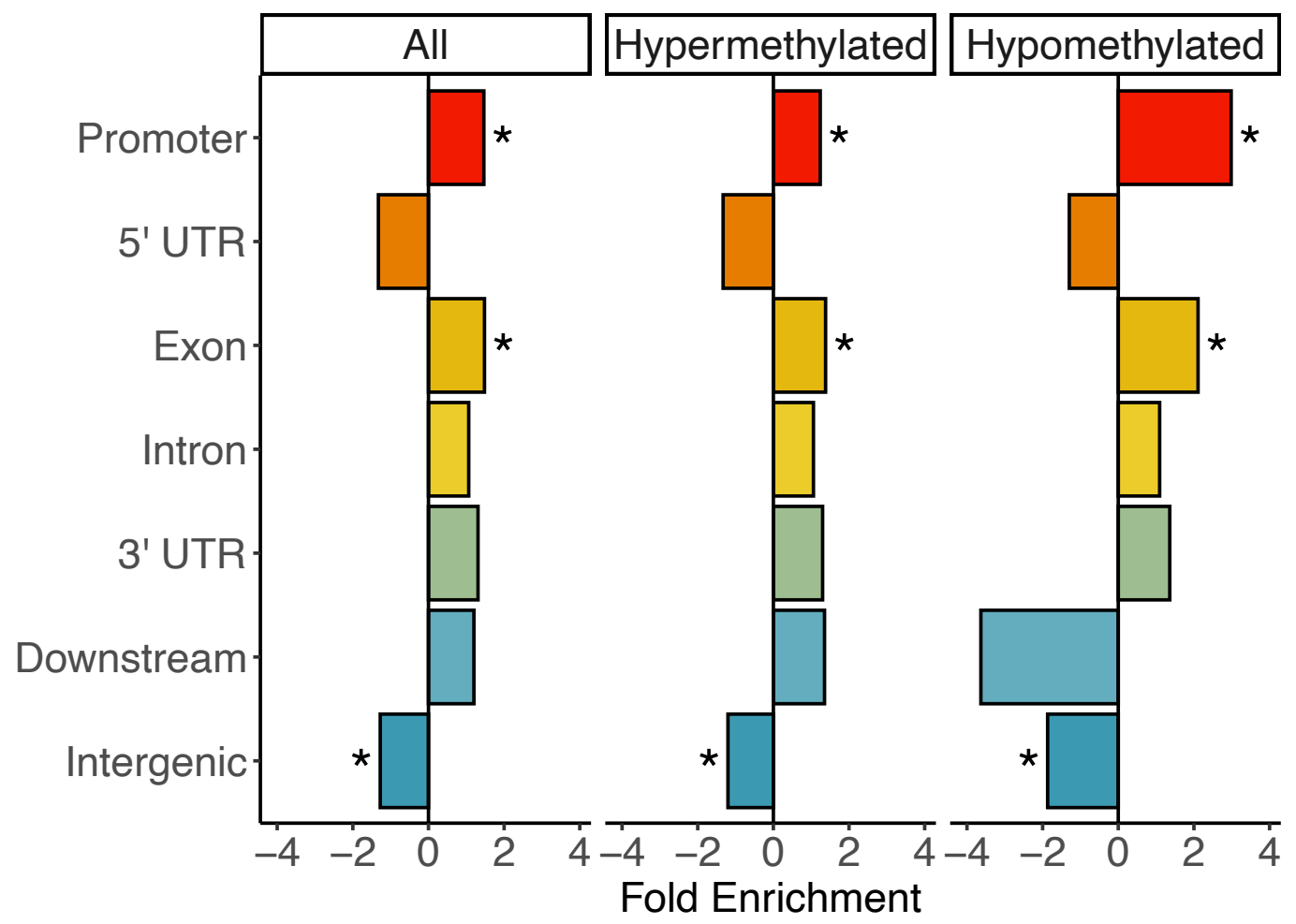

Supplemntary Figure 3 
A

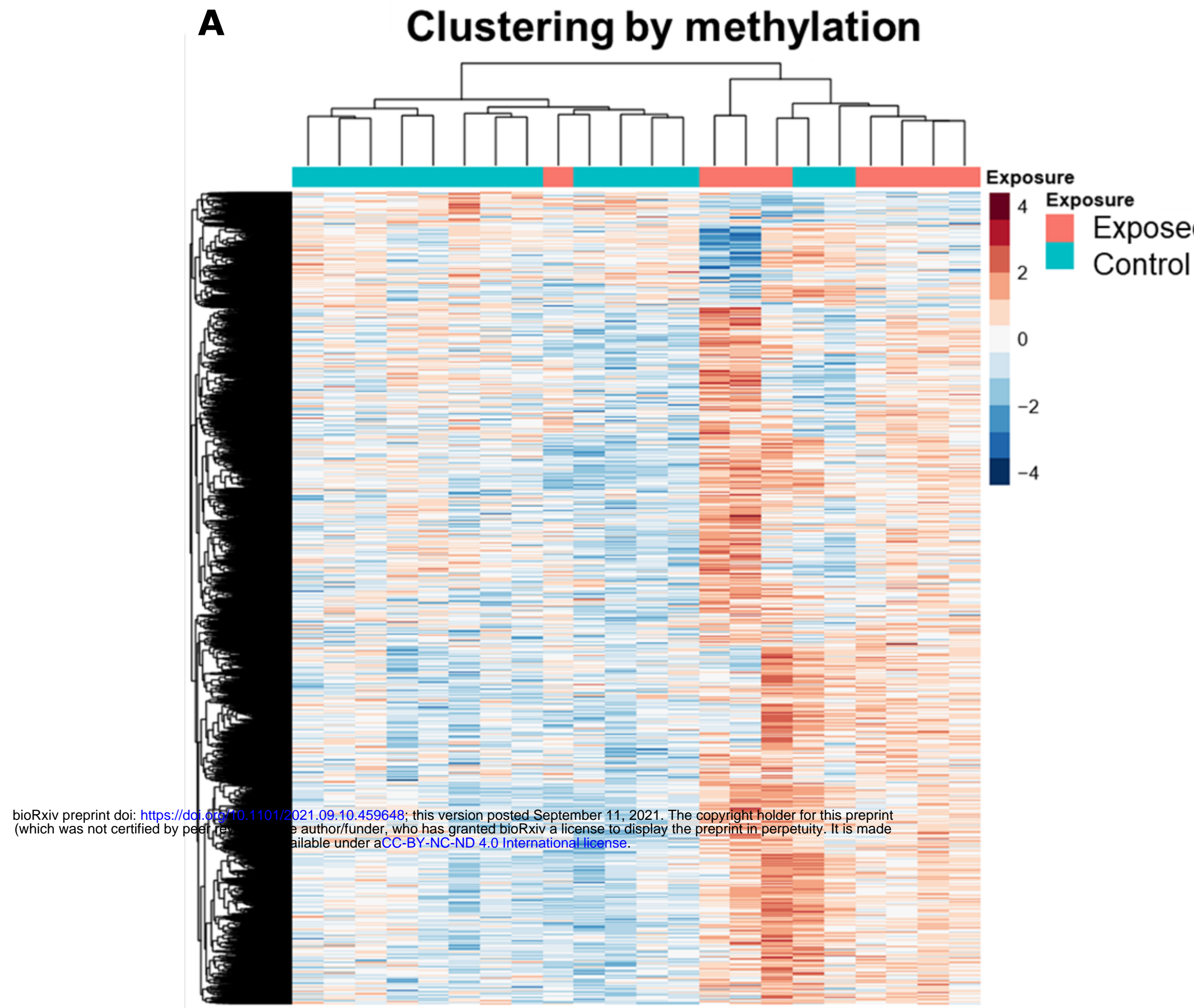

C

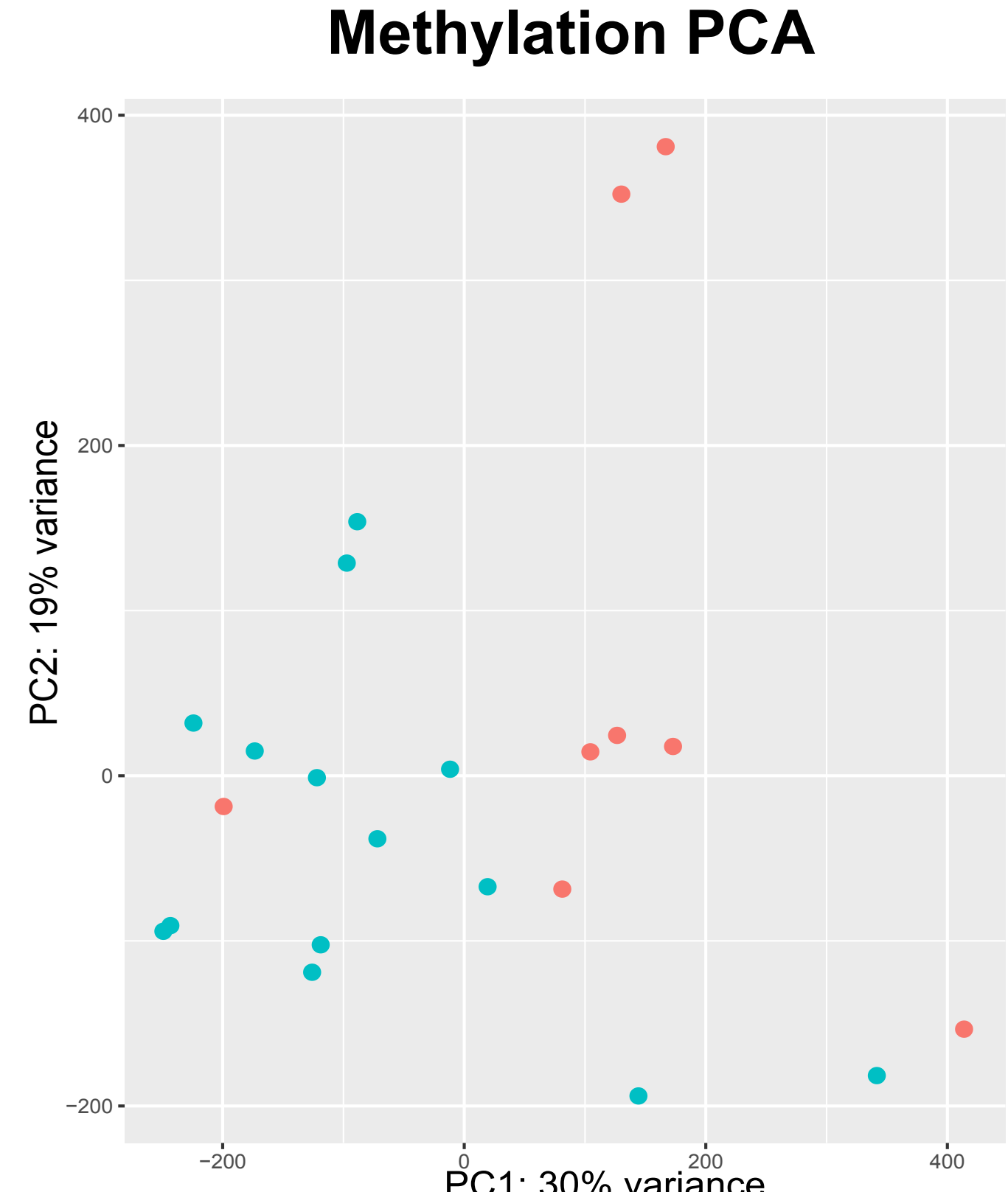

PC1: $30 \%$ variance
B Clustering by gene expression

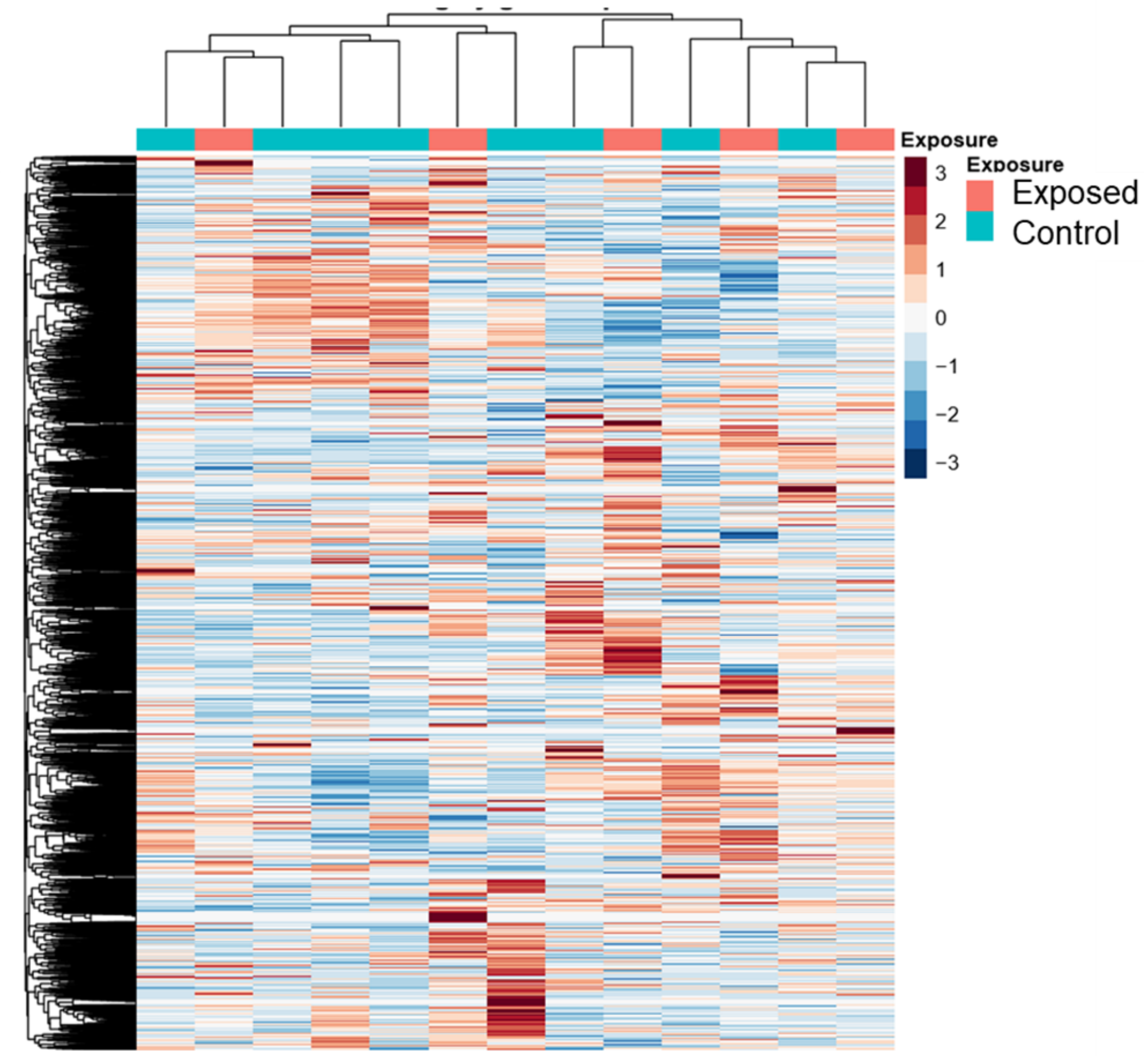

D

\section{Expression PCA}

\section{Exposure} - Exposed
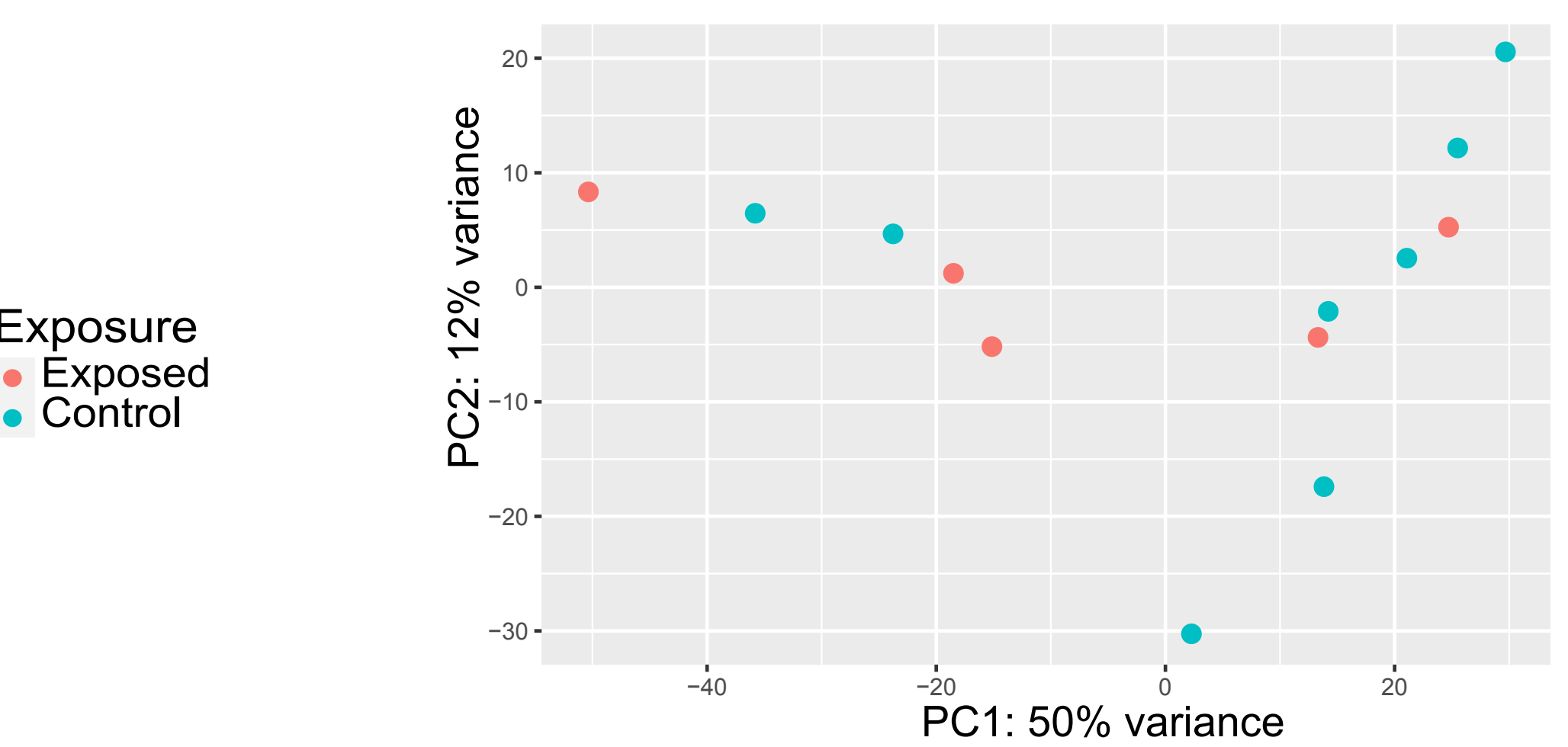

Exposure - Exposed - Control 


\section{Module-trait relationships}

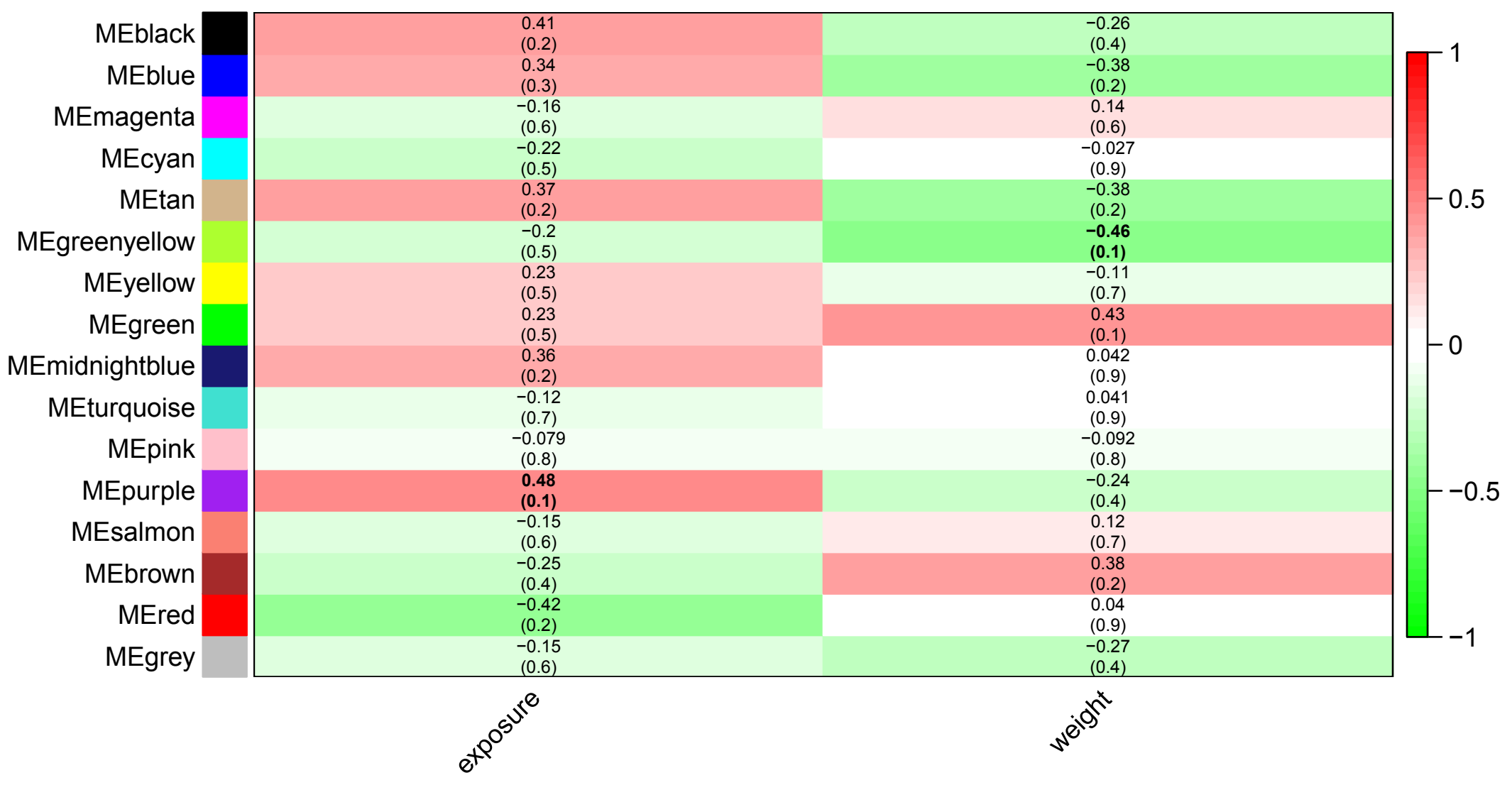

Supplemntary Figure 5 


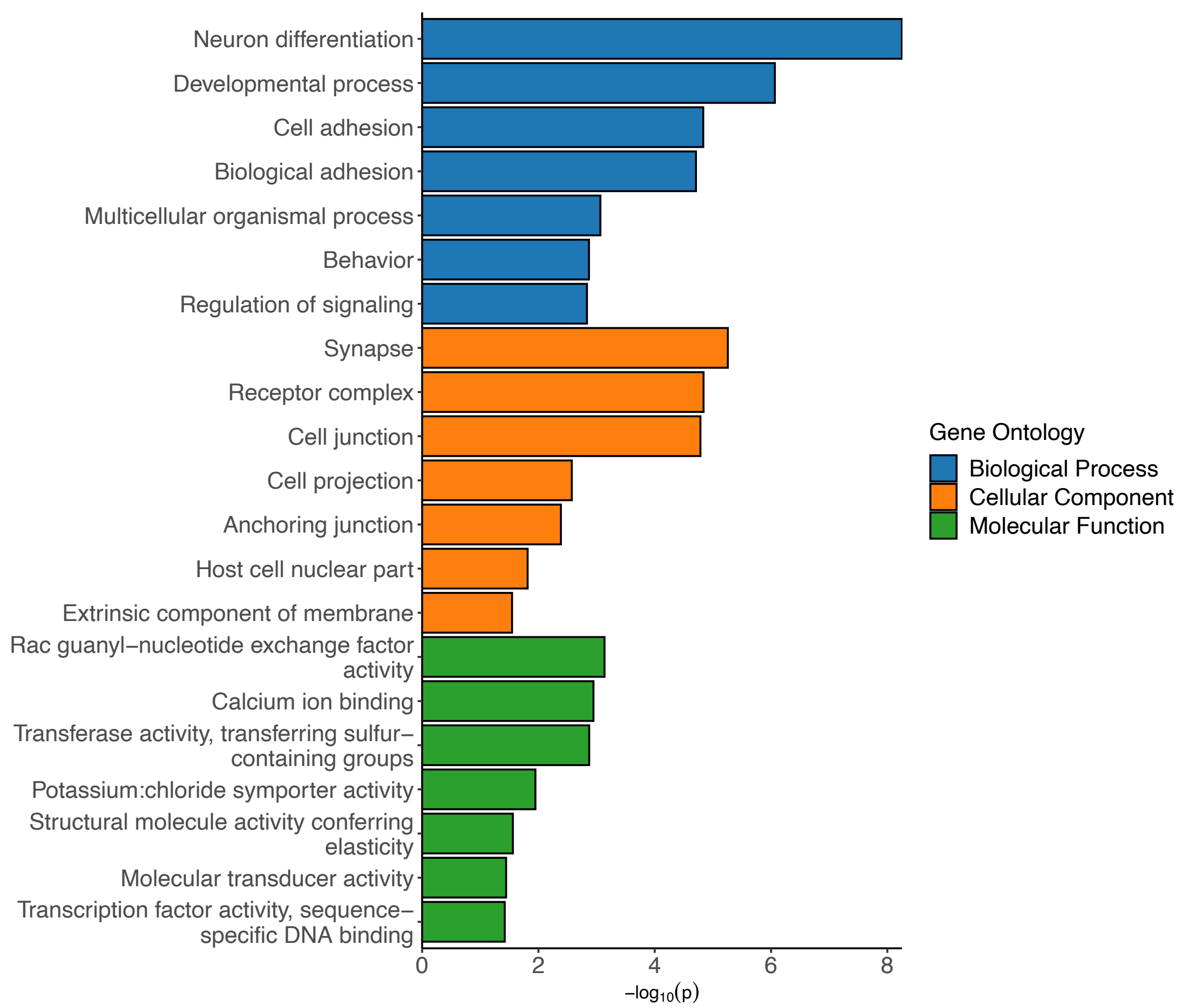

Supplemntary Figure 6 